This is the peer reviewed version of the following article: E. Oñate, A. Valls and J. García-Espinosa, Computation of turbulent flows using a finite calculus-finite element formulation, Int. J. Numer. Meth. Fluids 2007; 54:609-637, which has been published in final form at DOI: 10.1002/fld.1476. This article may be used for non-commercial purposes in accordance with Wiley Terms and Conditions for Use of Self-Archived Versions.

\title{
Computation of turbulent flows using a finite calculus-finite element formulation
}

\author{
E. Oñate ${ }^{* \dagger}$, A. Valls and J. García \\ International Center for Numerical Methods in Engineering (CIMNE), Technical University of Catalonia (UPC), \\ Gran Capitán s/n, 08034 Barcelona, Spain
}

\begin{abstract}
SUMMARY
We present a formulation for analysis of turbulent incompressible flows using a stabilized finite element method (FEM) based on the finite calculus (FIC) procedure. The stabilization terms introduced by the FIC approach allow to solve a wide range of fluid flow problems at different Reynolds numbers, including turbulent flows, without the need of a turbulence model. Examples of application of the FIC/FEM formulation to the analysis of 2D and 3D incompressible flows at large Reynolds numbers exhibiting turbulence features are presented.
\end{abstract}

KEY WORDS: turbulent flows; finite calculus; finite element method

\section{INTRODUCTION}

Stabilized finite element method (FEM) have been successfully used in the past to solve a wide range of fluid mechanics problems [1-28]. The intrinsic dissipative properties of the stabilization terms (which can be interpreted as an additional viscosity) typically suffice to yield good results for low and moderate values of the Reynolds number $(R e)$. For high values of $R e$ most stabilized FEM fail to provide physically sound results and the numerical solution is often unstable or inaccurate. The introduction of a turbulence model is then mandatory in order to obtain meaningful results.

The relationship between the dissipation introduced by a turbulence model and the intrinsic dissipative properties of stabilized FEM is an open topic which is attracting increasing attention in the CFD community [29-34]. It is clear that both remedies (the turbulence model and the

\footnotetext{
${ }^{*}$ Correspondence to: E. Oñate, International Center for Numerical Methods in Engineering (CIMNE), Technical University of Catalonia (UPC), Gran Capitán s/n, 08034 Barcelona, Spain.

†E-mail: onate@cimne.upc.edu

Contract/grant sponsor: Publishing Arts Research Council; contract/grant number: 98-1846389

Contract/grant sponsor: Consolider Programme of the Ministerio de Educación y Ciencia of Spain
} 
stabilization terms) play a similar role in the numerical solution, i.e. that of ensuring a solution which is 'physically correct' and as accurate as possible.

This paper extends the work recently presented by Oñate et al. $[35,36]$ where an enhanced stabilized FEM for incompressible flows was derived via finite calculus (FIC). The FIC approach is based on expressing the balance laws in mechanics in a domain of finite size. This introduces additional terms in the classical differential equations of momentum and mass balance of infinitesimal fluid mechanics [37-39]. The FIC terms are a function of characteristic length dimensions related to the finite element sizes and also to the values of the numerical solution. The FIC terms in the modified governing equations provide the necessary stabilization to the discrete equations obtained via the standard Galerkin FEM. The resulting FIC/FEM formulation allows to use loworder finite elements (such as linear triangles and tetrahedra) with an equal order approximation for the velocity and the pressure variables [35-39].

This paper shows that the nonlinear stabilization terms introduced by the FIC formulation into the momentum equations have a form of a nonlinear viscosity which is a function of the velocity and the velocity gradients. On the other hand, the FIC formulation introduces a Laplacian of pressure term into the mass balance equation. The resulting FIC/FEM formulation can be used to solve accurately high Re number flows without the need of introducing any turbulence model. The good results obtained in the examples presented indicate that the (nonlinear) FIC stabilization terms play the role of a turbulent model. The remarkable aspect of this approach is that the FIC equations are derived from basic principles in mechanics, such as baiance of momentum and mass over a domain of finite provide a very simple

The outline of the method are outlined the FIC governing equations to
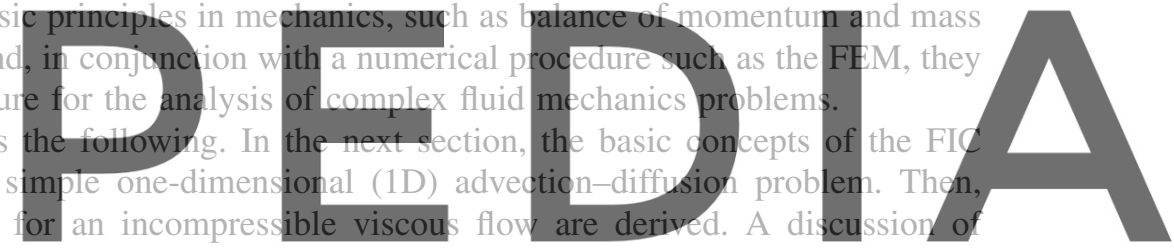

the stabilization terms introduced by the FIC procedure into the momentum and mass balance

flow analysis is detailed. The time integration of the discretized equations using a fractional step scheme is described. The procedure for computing the stabilization parameters is presented. The accuracy of the FIC/FEM formulation for analysis of turbulent flows is verified in two examples of application.

\section{FINITE CALCULUS: BASIC CONCEPTS}

The FIC method developed by Oñate and co-workers [35-57] is a consistent procedure for reformulating the governing equation in mechanics introducing new terms involving characteristic space and time dimensions into the equations. The modified equations are derived by invoking the balance laws in mechanics in a space-time domain of finite size. The new terms introduced by the FIC approach are essential to obtain physical (stable) numerical solutions for all ranges of the parameters governing the physical problem.

The merit of the modified equations via the FIC approach is that they lead to stabilized schemes using any numerical method. Moreover, many stabilized numerical methods typically used in practice can be recovered using the FIC equations [37, 43]. 


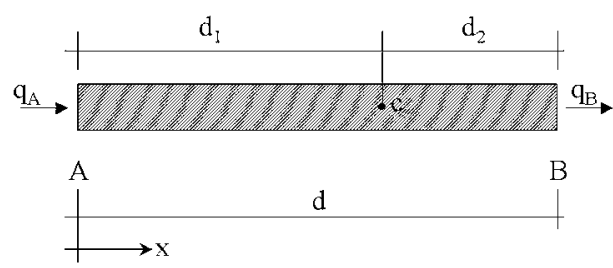

Figure 1. Equilibrium of fluxes in a space balance domain of finite size.

The FIC/FEM formulation has proven to be very effective for the solution of a wide class of problems, such as convection-diffusion [37-45] and convection-diffusion-reaction problems [46-48] involving arbitrary high gradients, incompressible flow problems accounting for free surface effects and fluid-structure interaction situations [49-54] and quasi and fully incompressible problems in solid mechanics [55-57].

In order to introduce the basic concepts of the FIC method, we will consider a steady-state convection-diffusion problem in a $1 \mathrm{D}$ domain $\Omega$ of length $L$. The equation of balance of fluxes in a subdomain of size $d$ belonging to $\Omega$ (Figure 1) is
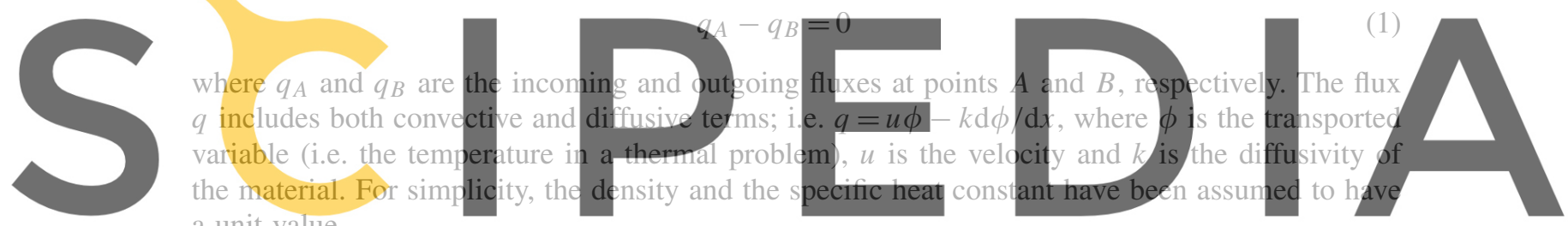

a unit value.

Let us express now the fluxes $q_{A}$ and $q_{B}$ in terms of the flux at an arbitrary point $C$ within

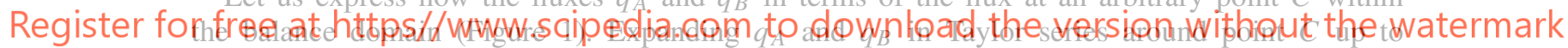
second-order terms gives

$$
q_{A}=q_{C}-\left.d_{1} \frac{\mathrm{d} q}{\mathrm{~d} x}\right|_{C}+\left.\frac{d_{1}^{2}}{2} \frac{\mathrm{d}^{2} q}{\mathrm{~d} x^{2}}\right|_{C}+O\left(d_{1}^{3}\right), \quad q_{B}=q_{C}+\left.d_{2} \frac{\mathrm{d} q}{\mathrm{~d} x}\right|_{C}+\left.\frac{d_{2}^{2}}{2} \frac{\mathrm{d}^{2} q}{\mathrm{~d} x^{2}}\right|_{C}+O\left(d_{2}^{3}\right)
$$

Substituting Equation (2) into Equation (1) gives after simplification

$$
\frac{\mathrm{d} q}{\mathrm{~d} x}-\frac{h}{2} \frac{\mathrm{d}^{2} q}{\mathrm{~d} x^{2}}=0
$$

where $h=d_{1}-d_{2}$ and all the derivatives are computed at the arbitrary point $C$.

Standard calculus theory assumes that the domain $d$ is of infinitesimal size and the resulting balance equation is simply $\mathrm{d} q / \mathrm{d} x=0$. We will relax this assumption and allow the space balance domain to have a finite size. The new balance equation (3) incorporates now the underlined term which introduces the characteristic length $h$.

Distance $h$ in Equation (3) is as a free parameter depending on the location of point $C$ within the balance domain. Note that $-d \leqslant h \leqslant d$ and, hence, $h$ can take a negative value. At the discrete solution level the domain $d$ should be replaced by the balance domain around a node. This gives for an equal size discretization $-l^{\mathrm{e}} \leqslant h \leqslant l^{\mathrm{e}}$ where $l^{\mathrm{e}}$ is the element or cell dimension. Equation (3) 
is the exact balance equation (up to second-order terms) for any 1D domain of finite size. The FIC balance equations can be used to derive numerical schemes with enhanced properties simply by computing the characteristic length parameter from an adequate 'optimality' rule, such as requiring an smaller error in the numerical solution [40-48].

Consider, for instance, the 1D convection-diffusion problem. Neglecting third-order derivatives of $\phi$, Equation (3) can be rewritten in terms of $\phi$ as

$$
-u \frac{\mathrm{d} \phi}{\mathrm{d} x}+\left(k+\frac{u h}{2}\right) \frac{\mathrm{d}^{2} \phi}{\mathrm{d} x^{2}}=0
$$

We see clearly that the FIC method introduces naturally an additional diffusion term in the standard convection-diffusion equation. This is the basis of the popular 'artificial diffusion' procedure $[1,2,10,25]$ where the characteristic length $h$ is typically expressed as a function of the cell or element dimension. The critical value of $h$ can be computed by requiring that the numerical solution of Equation (4) is physically meaningful [1,2, 10, 25, 37-43].

Equation (3) can be extended to account for source terms. The resulting FIC balance equation can then be written in compact form as [37]
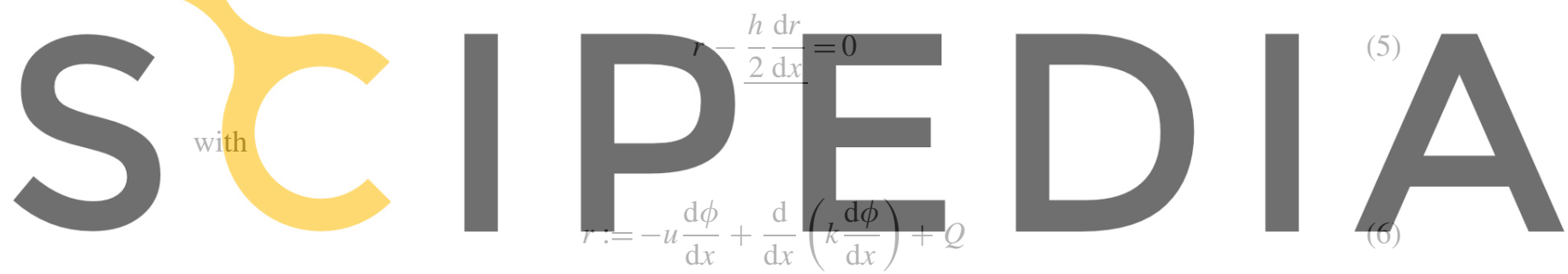

Register for free at https//www.scipedia.com to download the version without the watermark where $Q$ is the external source. Note that for $h=0$ the standard heat balance equation of the infinitesimal theory $(r=0)$ is recovered.

The essential (Dirichlet) boundary condition for Equation (5) is $\phi=\bar{\phi}$ on $\Gamma_{\phi}$ where $\Gamma_{\phi}$ is the boundary where the prescribed value $\bar{\phi}$ is imposed. For consistency, a stabilized Neumann boundary condition must be obtained as described next.

Let us consider a balance domain next to a Neumann boundary point B (Figure 2).

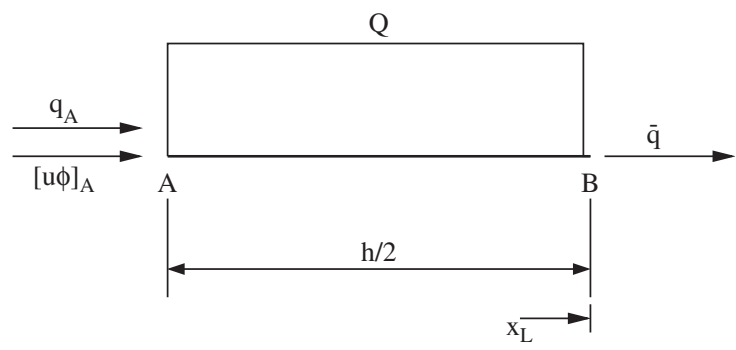

Figure 2. Balance domain next to a Neumann boundary point $B$. 
The length of the balance segment $A B$ next to a Neumann boundary is taken as one half of the characteristic length $h$ for the interior domain. The balance equation, assuming a constant distribution for the source $Q$, is

$$
\bar{q}-q\left(x_{A}\right)-[u \phi]_{A}-\frac{h}{2} Q=0
$$

where $\bar{q}$ is the prescribed total flux at $x=L$ and $x_{A}=x_{B}-h / 2$.

Using a second-order expansion for the advective and diffusive fluxes at point $A$ gives [37]

$$
-u \phi+k \frac{\mathrm{d} \phi}{\mathrm{d} x}+\bar{q}-\underline{\frac{h}{2}} r \quad \text { on } x=L
$$

where $r$ is given by Equation (6). Again for $h=0$, the infinitesimal form of the 1D Neumann boundary condition is obtained.

It is important to recall that the underlined terms in Equations (5) and (8) introduce the necessary stabilization in the discrete solution using whatever numerical scheme $[37,39]$.

Quite generally the FIC equations can be written for any problem in mechanics as

$$
r_{i}-\frac{h_{i j}}{2} \frac{\partial r_{i}}{\partial x_{j}}-\frac{\delta}{2} \frac{\partial r_{i}}{\partial t}=0, \quad i=1, n_{\mathrm{b}}, \quad j=1, n_{\mathrm{d}}
$$

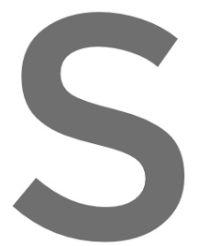

where $r_{i}$ is the $i$ th sta length parameters, $\delta$ is of balance equations problems). The usual specified. In this work, the term involving the
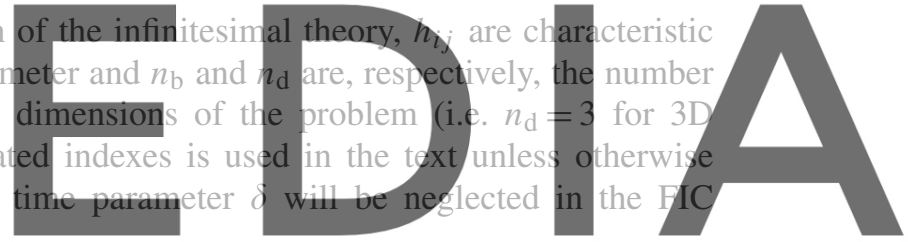

Register for free at https//www.scipedia.com to download the version without the watermark 3. FIC EQUATIONS FOR AN INCOMPRESSIBLE VISCOUS FLOW

The FIC momentum equations are obtained by expressing the balance of momentum along each of the space directions in a domain of 'finite' size. Figure 3 shows a typical finite domain for a two-dimensional (2D) problem. Following a procedure analogous to that explained in the previous
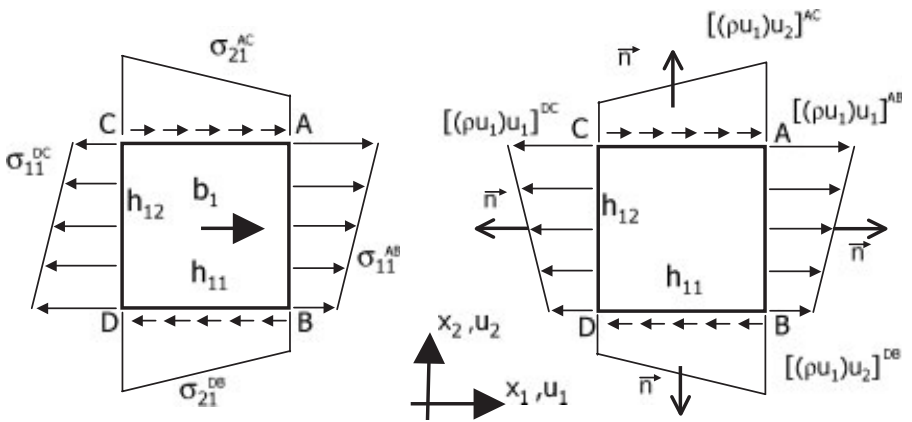

Figure 3. Finite domain where balance of momentum is imposed along the horizontal direction. 

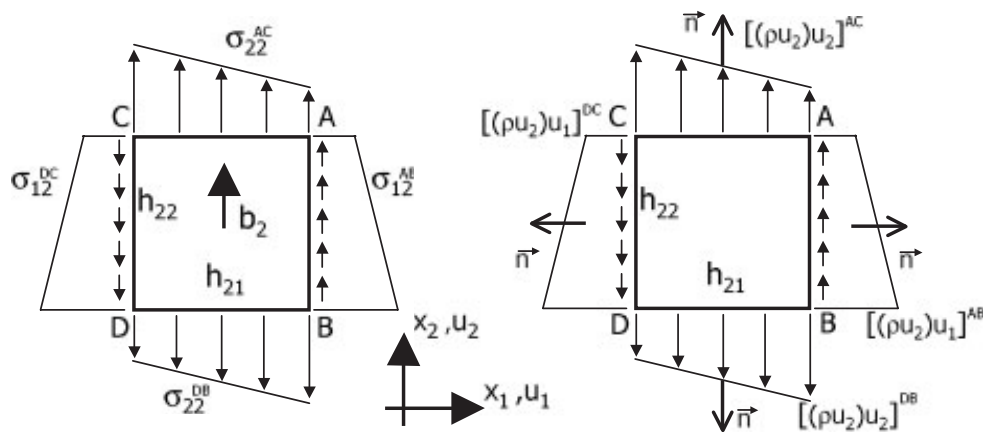

Figure 4. Finite domain where balance of momentum is imposed along the vertical direction.

section for the 1D advection-diffusion problem the balance equation along the $i$ th space direction can be written as

$$
\sum f_{i} \mathrm{~d} \Omega=\frac{\partial}{\partial t} \int_{\Omega} \rho u_{i} \mathrm{~d} \Omega+\int_{\Gamma}\left(\rho u_{i}\right) \mathbf{u}^{\mathrm{T}} \mathbf{n} \mathrm{d} \Gamma, \quad i=1, n_{\mathrm{d}}
$$

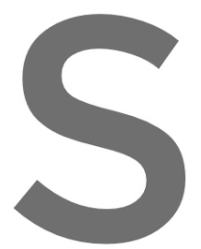

where $\rho$ is the density,

velocity along the $i$ th $\mathrm{sp}$

normal to the domain bor

of the balance domain a

Expressing the values

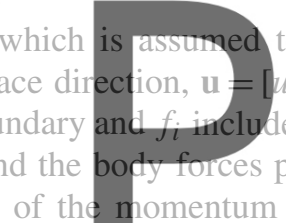

be
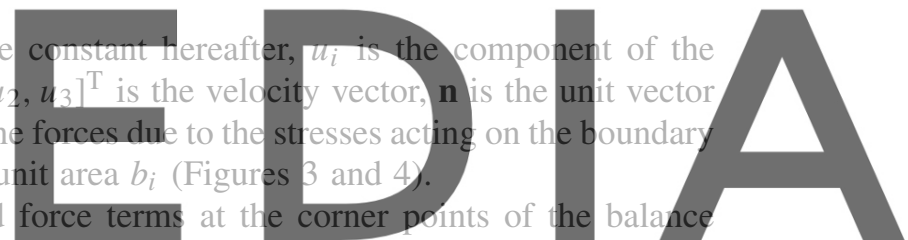

domain in terms of the values at the corner point A using higher-order Taylor expansions in the

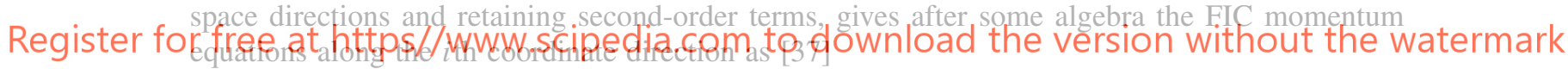

$$
\bar{r}_{m_{i}}-\underline{\frac{1}{2} h_{i j} \frac{\partial \bar{r}_{m_{i}}}{\partial x_{j}}}=0, \quad i, j=1, n_{\mathrm{d}}
$$

where

$$
\bar{r}_{m_{i}}:=\rho\left[\frac{\partial u_{i}}{\partial t}+\frac{\partial}{\partial x_{j}}\left(u_{i} u_{j}\right)\right]-\frac{\partial \sigma_{i j}}{\partial x_{j}}-b_{i}
$$

with $\sigma_{i j}=s_{i j}-p \delta_{i j}$, where $p$ is the pressure, $\delta_{i j}$ is the Dirac delta and $s_{i j}$ are the viscous stresses related to the velocities by the standard expression

$$
s_{i j}=2 \mu\left(\varepsilon_{i j}-\delta_{i j} \frac{1}{3} \frac{\partial u_{k}}{\partial x_{k}}\right)
$$

where

$$
\varepsilon_{i j}=\frac{1}{2}\left(\frac{\partial u_{i}}{\partial x_{j}}+\frac{\partial u_{j}}{\partial x_{i}}\right)
$$




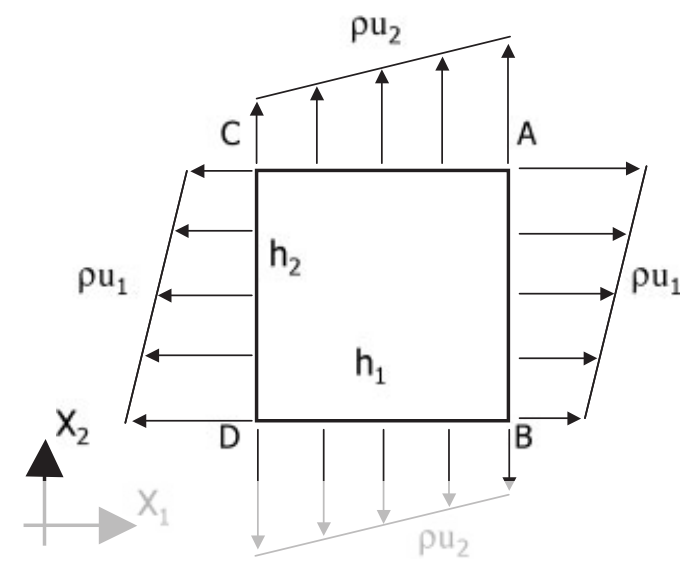

Figure 5. Finite domain where balance of mass is enforced.

Note that distance $h_{12}$ is arbitrary when writing the balance of momentum along the $x_{1}$ direction. The same applies for the distance $h_{21}$ when deriving the balance ququation along the $x_{2}$ direction. Thus, in general, $h_{12}$

The convective term form, as deduced from be written by introducin of $\bar{r}_{m_{i}}$ and using the giving

\section{Register for free at https//www.scipedia.com to download the version without the watermark}

$$
r_{m_{i}}-\frac{-}{2} h_{i j} \frac{\partial m_{i}}{\partial x_{j}}=0, \quad i, j=1, n_{\mathrm{d}}
$$

with

$$
r_{m_{i}}:=\rho\left(\frac{\partial u_{i}}{\partial t}+u_{j} \frac{\partial u_{i}}{\partial x_{j}}\right)+\frac{\partial p}{\partial x_{i}}-\frac{\partial s_{i j}}{\partial x_{j}}-b_{i}
$$

The two forms of the FIC balance equations (11) and (15) are identical for the exact incompressible solution. Both forms will be used to the advantage of each derivation step in the following sections.

\subsection{Mass balance equation}

The FIC mass balance equation is obtained by invoking the balance of mass in the finite domain of Figure 5

$$
\int_{\Gamma} \rho \mathbf{u}^{\mathrm{T}} \mathbf{n} \mathrm{d} \Gamma=0
$$


Expanding the values of $\rho u_{i}$ at the corner points in terms of the value at the corner point $A$ gives the FIC mass balance equation as [37,38]

$$
\varepsilon_{v}-\underline{\frac{1}{2}} h_{j} \frac{\partial \varepsilon_{v}}{\partial x_{j}}=0, \quad j=1, n_{\mathrm{d}}
$$

with

$$
\varepsilon_{v}=\frac{\partial u_{i}}{\partial x_{i}}
$$

Note that a matrix form of the characteristic distances is not obtained in this case as the mass balance equation expresses the conservation of the mass in the domain $A B C D$ of Figure 5 with dimensions $h_{1}$ and $h_{2}$. Distances $h_{1}$ and $h_{2}$ are in general different from the distances $h_{i j}$ defining the domain where balance of momentum is enforced. In the following, we will assume that $h_{1}=h_{11}$ and $h_{2}=h_{22}$ for simplicity.

\subsection{Boundary conditions}

The FIC Neumann boundary conditions are obtained by expressing the balance of momentum in a domain of finite size adjacent to a boundary $\Gamma_{t}$ where the surface tractions $t_{i}$ act. After some
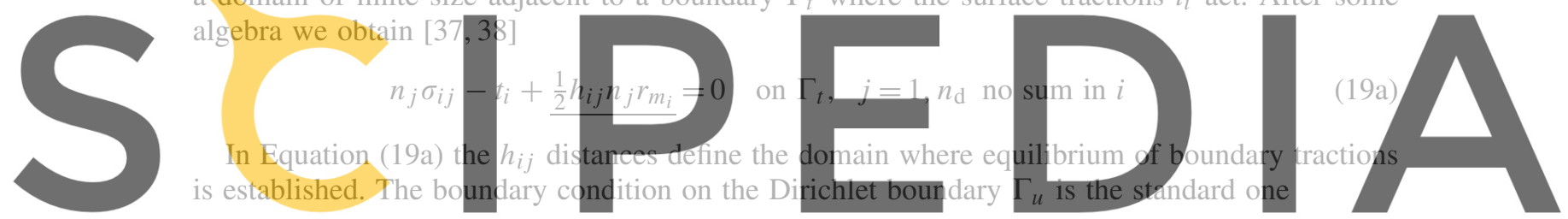

$$
u_{j}-u_{j}^{p}=0 \quad \text { on } \Gamma_{u}
$$

(19b)

Register for free at https/fwww.scipedia.com to download the version without the watermark element dimensions. The infinitesimal form of the fluid mechanics equations is recovered by making these distances equal to zero.

Equations (11)-(19) are the starting points for deriving stabilized FEM for solving the incompressible Navier-Stokes equations. The underlined FIC terms in Equations (11) (or (15)) and (19a) are essential to overcome the numerical instabilities due to the convective terms in the momentum equations, whereas the underlined terms in Equation (18a) take care of the instabilities due to the incompressibility constraint. An important feature of the FIC formulation is that it allows to use equal order interpolation for the velocity and pressure variables [38, 54].

\section{A DISCUSSION OF THE STABILIZATION TERMS IN THE FIC EQUATIONS}

The compact residual forms of the FIC equations of momentum balance (Equations (11) or (15)) and mass balance (Equation (18a)) hide the relevant terms that contribute to the stabilization of the numerical solution for all flow regimes. We will show next that the FIC terms introduce a nonlinear anisotropic viscosity into the standard momentum equations of the infinitesimal theory. Also, it is shown that the FIC terms in the mass balance equation introduce a pressure Laplacian term. 


\subsection{Momentum equations}

Let us write the $i$ th FIC momentum equation of Equation (15) as

$$
\rho\left[\frac{\partial u_{i}}{\partial t}+u_{j} \frac{\partial u_{i}}{\partial x_{j}}\right]+\frac{\partial p}{\partial x_{i}}-\frac{\partial s_{i j}}{\partial x_{j}}-b_{i}-\frac{h_{i k}}{2} \frac{\partial}{\partial x_{k}}\left[c_{i}+\rho u_{j} \frac{\partial u_{i}}{\partial x_{j}}\right]=0
$$

where

$$
c_{i}:=\rho \frac{\partial u_{i}}{\partial t}-\frac{\partial \sigma_{i j}}{\partial x_{j}}-b_{i}
$$

is termed the convective projection term. Note that in the infinitesimal limit

$$
r_{m_{i}}:=c_{i}+\rho u_{j} \frac{\partial u_{i}}{\partial x_{j}}=0
$$

Substituting the expression of the viscous stresses of Equation (13) into Equation (20) and using Equation (14) yields after small algebra
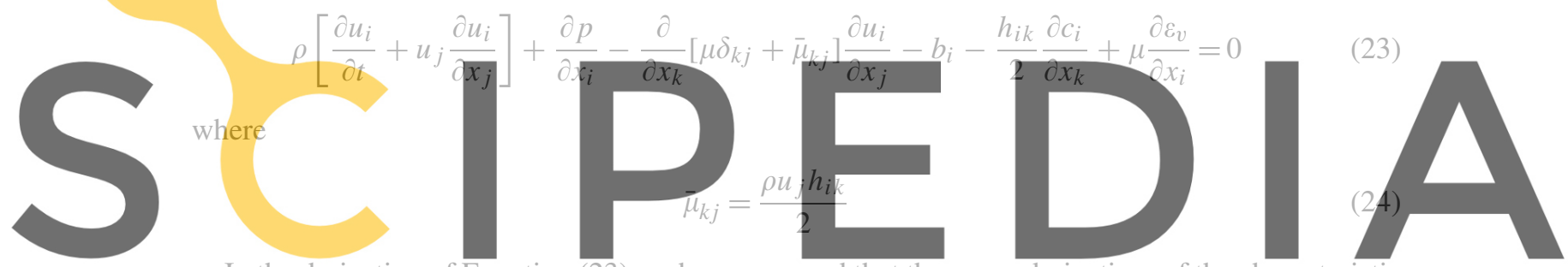

In the derivation of Equation (23) we have assumed that the space derivatives of the characteristic

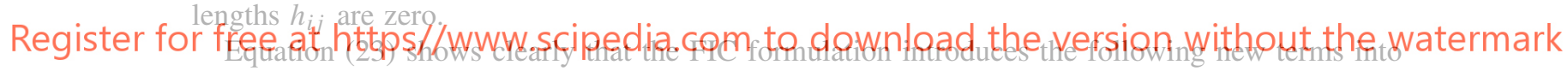

the $i$ th momentum equation of the infinitesimal theory:

(a) an additional (nonlinear) anisotropic viscosity $\bar{\mu}_{k j}$ given by Equation (24) and

(b) a convective projection term of value $-h_{i k} / 2\left(\partial c_{i} / \partial x_{k}\right)$.

The last term in Equation (23) involving $\varepsilon_{v}$ is usually disregarded in practice. We have found, however, that retaining this term is very important in free surface viscous flows [58]. For this reason, the full compact (residual) form of Equations (11) or (15) is used in practice.

\subsection{Mass balance equation}

The FIC momentum balance equation (11) is written as (assuming the viscosity $\mu$ to be constant)

$$
\rho\left[\frac{\partial u_{i}}{\partial t}+u_{j} \frac{\partial u_{i}}{\partial x_{j}}\right]+\rho u_{i} \varepsilon_{v}+\frac{\partial p}{\partial x_{i}}-\frac{\partial}{\partial x_{j}}\left(2 \mu \varepsilon_{i j}\right)+\frac{2}{3} \mu \frac{\partial \varepsilon_{v}}{\partial x_{i}}-b_{i}-\frac{h_{i j}}{2} \frac{\partial \bar{r}_{m_{i}}}{\partial x_{j}}=0
$$

From the FIC mass balance equation (18a) we deduce

$$
\varepsilon_{v}=\frac{h_{j}}{2} \frac{\partial \varepsilon_{v}}{\partial x_{j}}
$$


Substituting $\varepsilon_{v}$ from Equation (26) into (25) gives after small algebra

$$
\frac{\partial \varepsilon_{v}}{\partial x_{i}}=\frac{1}{a_{i}}\left[\frac{h_{i j}}{2} \frac{\partial \bar{r}_{m_{i}}}{\partial x_{j}}-r_{m_{i}}-\rho \frac{u_{i} h_{k}}{2} \frac{\partial \varepsilon_{v}}{\partial x_{k}}\right], \quad i \neq k
$$

with

$$
a_{i}=\frac{2 \mu}{3}+\rho \frac{u_{i} h_{i}}{2} \text { no sum in } i
$$

We note that all terms in Equation (27) vanish for the exact solution. On this basis, the following simplified expression is chosen for $\partial \varepsilon_{v} / \partial x_{i}$ :

$$
\frac{\partial \varepsilon_{v}}{\partial x_{i}}=\frac{h_{i i}}{2 a_{i}} \frac{\partial r_{m_{i}}}{\partial x_{i}} \text { no sum in } i
$$

Substituting Equation (29) into (18a) gives the following useful expression for the FIC mass balance equation incorporating the momentum equations $r_{m_{i}}$ :
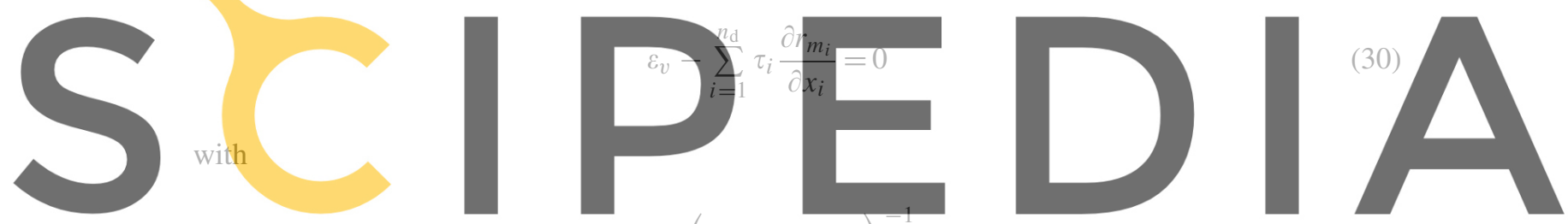

\section{Register for free at https//www.scipedia.con to diownload the version without the watermark}

Note that in Equation (31) the assumption $h_{i}=h_{i i}$ has been used.

The $\tau_{i}$ 's in Equation (33) when multiplied by the density are equivalent to the intrinsic time parameters, seen extensively in the stabilization literature [1-28]. The interest of Equation (30) is that it introduces a Laplacian of pressure term into the mass balance equations through the first derivative of $r_{m_{i}}$. To show this clearly it is convenient to express the $r_{m_{i}}$ terms as

$$
r_{m_{i}}=\frac{\partial p}{\partial x_{i}}+\pi_{i}
$$

where $\pi_{i}$ are termed the pressure gradient projections. The exact expression of $\pi_{i}$ is deduced by substracting the pressure gradient terms from the standard momentum equations, i.e.

$$
\pi_{i}:=\rho\left[\frac{\partial u_{i}}{\partial t}+u_{j} \frac{\partial u_{i}}{\partial x_{j}}\right]-\frac{\partial}{\partial x_{j}}\left(2 \mu s_{i j}\right)-b_{i}
$$

This form of $\pi_{i}$ will not be, however, used in practice as the nodal values of $\pi_{i}$ are directly computed from the projection of the pressure gradients, as explained in the next section. 
Substituting the expression of $r_{m_{i}}$ of Equation (32) into Equation (30) gives

$$
\varepsilon_{v}-\sum_{i=1}^{n_{\mathrm{d}}} \tau_{i} \frac{\partial}{\partial x_{i}}\left(\frac{\partial p}{\partial x_{i}}+\pi_{i}\right)=\varepsilon_{v}-\sum_{i=1}^{n_{\mathrm{d}}} \tau_{i}\left[\frac{\partial^{2} p}{\partial x_{i}^{2}}+\frac{\partial \pi_{i}}{\partial x_{i}}\right]=0
$$

Equation (33) shows that the FIC formulation introduces naturally a Laplacian of pressure term into the mass balance equation. The consistency of the approach is ensured by the pressure gradient projection terms $\pi_{i}$, as the bracketed terms in Equation (34) are equal to the momentum equations and, therefore, they vanish as these equations are satisfied for the 'exact' solution.

\section{INTEGRAL FORM OF THE FIC GOVERNING EQUATIONS}

The weighted residual form of the momentum and mass balance equations (Equations (15) and (30)) is

$$
\begin{gathered}
\int_{\Omega} \delta u_{i}\left[r_{m_{i}}-\frac{h_{i j}}{2} \frac{\partial r_{m_{i}}}{\partial x_{j}}\right] \mathrm{d} \Omega+\int_{\Gamma_{t}} \delta u_{i}\left(\sigma_{i j} n_{j}-t_{i}+\frac{h_{i j}}{2} n_{j} r_{m_{i}}\right) \mathrm{d} \Gamma=0 \\
\int_{\Omega} q\left[\varepsilon_{v}-\sum_{i=1}^{n_{\mathrm{d}}} \tau_{i} \frac{\partial r_{m_{i}}}{\partial x_{i}}\right] \mathrm{d} \Omega=0
\end{gathered}
$$

where $\delta u_{i}$ and $q$ are arbitrary weighting functions representing virtual velocities and virtual pressure fields. Integrating by parts the terms involving the derivatives of $r_{m_{i}}$ in Equations (34) gives

$$
\begin{aligned}
& \int_{\Omega} \delta u_{i} r_{m_{i}} \mathrm{~d} \Omega+\int_{\Gamma_{t}} \delta u_{i}\left(\sigma_{i j} n_{j}-t_{i}\right) \mathrm{d} \Gamma+\int_{\Omega} \frac{h_{i j}}{2} \frac{\partial \delta u_{i}}{\partial x_{j}} r_{m_{i}} \mathrm{~d} \Omega=0 \\
& \int_{\Omega} q \varepsilon_{v} \mathrm{~d} \Omega+\int_{\Omega}\left[\sum_{i=1}^{n_{\mathrm{d}}} \tau_{i} \frac{\partial q}{\partial x_{i}} r_{m_{i}}\right] \mathrm{d} \Omega-\int_{\Gamma}\left[\sum_{i=1}^{n_{\mathrm{d}}} q \tau_{i} n_{i} r_{m_{i}}\right] \mathrm{d} \Gamma=0
\end{aligned}
$$

We will neglect hereonwards the third integral in Equation (36b) by assuming that $r_{m_{i}}$ is negligible on the boundaries. The deviatoric stresses and the pressure terms in the first integral of Equation (36a) are integrated by parts in the usual manner. The resulting momentum and mass balance equations are

$$
\begin{gathered}
\int_{\Omega}\left[\delta u_{i} \rho\left(\frac{\partial u_{i}}{\partial t}+u_{j} \frac{\partial u_{i}}{\partial x_{j}}\right)+\frac{\partial \delta u_{i}}{\partial x_{j}}\left(s_{i j}-\delta_{i j} p\right)\right] \mathrm{d} \Omega-\int_{\Omega} \delta u_{i} b_{i} \mathrm{~d} \Omega \\
-\int_{\Gamma_{t}} \delta u_{i} t_{i} \mathrm{~d} \Gamma+\int_{\Omega} \frac{h_{i j}}{2} \frac{\partial \delta u_{i}}{\partial x_{j}} r_{m_{i}} \mathrm{~d} \Omega=0 \\
\int_{\Omega} q \frac{\partial u_{i}}{\partial x_{i}} \mathrm{~d} \Omega+\int_{\Omega}\left[\sum_{i=1}^{n_{\mathrm{d}}} \tau_{i} \frac{\partial q}{\partial x_{i}} r_{m_{i}}\right] \mathrm{d} \Omega=0
\end{gathered}
$$


The computation of the residual terms is simplified if we introduce the convective projections $c_{i}$ (Equation (22)) and the pressure gradient projections $\pi_{i}$ (Equation (32)). We therefore express $r_{m_{i}}$ in Equations (37a) and (37b) in terms of $c_{i}$ and $\pi_{i}$, respectively, which then become additional variables. The system of integral equations is now augmented in the necessary number of equations by imposing that the residual $r_{m_{i}}$ vanishes (in a weighted residual sense) for both forms given by Equations (22) and (32). This gives the final system of governing equation as

$$
\begin{gathered}
\int_{\Omega}\left[\delta u_{i} \rho\left(\frac{\partial u_{i}}{\partial t}+u_{j} \frac{\partial u_{i}}{\partial x_{j}}\right)+\frac{\partial \delta u_{i}}{\partial x_{j}}\left(s_{i j}-\delta_{i j} p\right)\right] \mathrm{d} \Omega-\int_{\Omega} \delta u_{i} b_{i} \mathrm{~d} \Omega \\
-\int_{\Gamma_{t}} \delta u_{i} t_{i} \mathrm{~d} \Gamma+\int_{\Omega} \frac{h_{i k}}{2} \frac{\partial\left(\delta u_{i}\right)}{\partial x_{k}}\left(\rho u_{j} \frac{\partial u_{i}}{\partial x_{j}}+c_{i}\right) \mathrm{d} \Omega=0 \\
\int_{\Omega} q \frac{\partial u_{i}}{\partial x_{i}} \mathrm{~d} \Omega+\int_{\Omega} \sum_{i=1}^{n_{\mathrm{d}}} \tau_{i} \frac{\partial q}{\partial x_{i}}\left(\frac{\partial p}{\partial x_{i}}+\pi_{i}\right) \mathrm{d} \Omega=0 \\
\int_{\Omega} \delta c_{i} \rho\left(\rho u_{j} \frac{\partial u_{i}}{\partial x_{j}}+c_{i}\right) \mathrm{d} \Omega=0 \text { no sum in } i \\
\int_{\Omega} \delta \pi_{i} \tau_{i}\left(\frac{\partial p}{\partial x_{i}}+\pi_{i}\right) \mathrm{d} \Omega=0 \text { no sum in } i
\end{gathered}
$$

with $i, j, k=1, n_{\mathrm{d}}$. In Equations (40) and (41) $\delta c_{i}$ and $\delta \pi_{i}$ are appropriate weighting functions and the $\rho$ and $\tau_{i}$ weights are introduced for convenience.

Accounting for the convective and pressure gradient projections enforces the consistency of the formulation as it ensures that the stabilization terms in Equations (38) and (39) have a residual form which vanishes for the 'exact' solution. Neglecting these terms can reduce the accuracy of the numerical solution and it makes the formulation more sensitive to the value of the stabilization parameters [54-56].

\section{FINITE ELEMENT DISCRETIZATION}

We choose $C^{\circ}$ continuous linear interpolations for the velocities, the pressure, the convective projections $c_{i}$ and the pressure gradient projections $\pi_{i}$ over 3-noded triangles (2D) and 4-noded tetrahedra (3D). The linear interpolations are written as

$$
\begin{array}{ll}
u_{i}=N^{k} \bar{u}_{i}^{k}, & p=N^{k} \bar{p}^{k} \\
c_{i}=N^{k} \bar{c}_{i}^{k}, & \pi_{i}=N^{k} \bar{\pi}_{i}^{k}
\end{array}
$$

where the sum goes over the number of nodes of each element $n=3,4$ for triangles/tetrahedra, $(\cdot)^{k}$ denotes the nodal variables and $N^{k}$ are the linear shape functions $[25,26]$. 
Substituting approximations (42) into Equations (38)-(41) and choosing the Galerkin form with $\delta u_{i}=q=\delta c_{i}=\delta \pi_{i}=N^{i}$ lead to the following system of discretized equations:

$$
\begin{gathered}
\mathbf{M} \dot{\overline{\mathbf{u}}}+\mathbf{H} \overline{\mathbf{u}}-\mathbf{G} \overline{\mathbf{p}}+\mathbf{C} \overline{\mathbf{c}}=\mathbf{f} \\
\mathbf{G}^{\mathrm{T}} \overline{\mathbf{u}}+\hat{\mathbf{L}} \overline{\mathbf{p}}+\mathbf{Q} \bar{\pi}=\mathbf{0} \\
\hat{\mathbf{C}} \overline{\mathbf{u}}+\mathbf{M} \overline{\mathbf{c}}=\mathbf{0} \\
\mathbf{Q}^{\mathrm{T}} \overline{\mathbf{p}}+\hat{\mathbf{M}} \bar{\pi}=\mathbf{0}
\end{gathered}
$$

where

$$
\mathbf{H}=\mathbf{A}+\mathbf{K}+\hat{\mathbf{K}}
$$

The matrices and vectors in the above equations are assembled from the element contributions in the standard manner. The element expressions for 3D problems are given next

$$
\begin{aligned}
& \mathbf{M}_{i j}^{\mathrm{e}}=\int_{\Omega^{\mathrm{e}}} \rho N^{i} N^{j} \mathbf{I}_{3} \mathrm{~d} \Omega, \quad \mathbf{C}_{i j}^{\mathrm{e}}=\frac{1}{2} \int_{\Omega^{\mathrm{e}}}\left[\begin{array}{ccc}
\mathbf{h}_{1}^{\mathrm{T}} \nabla N^{i} & 0 & 0 \\
0 & \mathbf{h}_{2}^{\mathrm{T}} \nabla N^{i} & 0 \\
0 & 0 & \mathbf{h}_{3}^{\mathrm{T}} \boldsymbol{\nabla} N^{i}
\end{array}\right] N^{j} \mathrm{~d} \Omega \\
& \mathbf{A}_{i j}^{\mathrm{e}}=\int_{\Omega^{\mathrm{e}}} \rho N^{i}\left(\mathbf{u}^{\mathrm{T}} \nabla N^{j}\right) \mathbf{I}_{3} \mathrm{~d} \Omega, \quad \mathbf{K}_{i j}^{\mathrm{e}}=\int_{\Omega^{\mathrm{e}}} \mathbf{B}_{i}^{\mathrm{T}} \mathbf{D} \mathbf{B}_{j} \mathrm{~d} \Omega, \quad \hat{\mathbf{K}}_{i j}^{\mathrm{e}}=\int_{\Omega^{\mathrm{e}}}\left(\bar{\nabla} N^{i}\right)^{\mathrm{T}} \overline{\mathbf{D}} \bar{\nabla} N^{j} \mathrm{~d} \boldsymbol{\Omega} \\
& \mathbf{G}_{i j}^{\mathrm{e}}=\int_{\Omega^{\mathrm{e}}} \mathbf{B}_{i}^{\mathrm{T}} \mathbf{m} N^{j} \mathrm{~d} \Omega, \quad \hat{L}_{i j}^{\mathrm{e}}=\int_{\Omega^{\mathrm{e}}}\left(\nabla N^{i}\right)^{\mathrm{T}}[\tau] \nabla N^{j} \mathrm{~d} \Omega \\
& \mathbf{Q}_{i j}^{\mathrm{e}}=\int_{\Omega^{\mathrm{e}}}\left(\nabla N^{i}\right)^{\mathrm{T}} N^{j}[\tau] \mathrm{d} \Omega, \quad \hat{\mathbf{C}}_{i j}=\int_{\Omega^{\mathrm{e}}} \rho^{2} N^{i}\left(\mathbf{u}^{\mathrm{T}} \nabla N^{j}\right) \mathbf{I}_{3} \mathrm{~d} \Omega \\
& \hat{\mathbf{M}}_{i j}=\int_{\Omega^{\mathrm{e}}} N^{i} N^{j}[\tau] \mathrm{d} \Omega, \quad \mathbf{m}=[1,1,1,0,0,0]^{\mathrm{T}}, \quad \mathbf{h}_{i}=\left[h_{i 1}, h_{i 2}, h_{i 3}\right]^{\mathrm{T}} \\
& \overline{\mathbf{D}}=\frac{\rho}{2}\left[\begin{array}{ccc}
\mathbf{h}_{1} \mathbf{u}^{\mathrm{T}} & \mathbf{0} & \mathbf{0} \\
\mathbf{0} & \mathbf{h}_{2} \mathbf{u}^{\mathrm{T}} & \mathbf{0} \\
\mathbf{0} & \mathbf{0} & \mathbf{h}_{3} \mathbf{u}^{\mathrm{T}}
\end{array}\right], \quad \bar{\nabla}=\left[\begin{array}{ccc}
\nabla & \mathbf{0} & \mathbf{0} \\
\mathbf{0} & \nabla & \mathbf{0} \\
\mathbf{0} & \mathbf{0} & \nabla
\end{array}\right] \\
& \nabla=\left\{\begin{array}{c}
\frac{\partial}{\partial x_{1}} \\
\frac{\partial}{\partial x_{2}} \\
\frac{\partial}{\partial x_{3}}
\end{array}\right\}, \quad[\tau]=\left[\begin{array}{ccc}
\tau_{1} & 0 & 0 \\
0 & \tau_{2} & 0 \\
0 & 0 & \tau_{3}
\end{array}\right]
\end{aligned}
$$




$$
\begin{aligned}
& \mathbf{B}_{i}=\left[\begin{array}{ccc}
\frac{\partial N^{i}}{\partial x_{1}} & 0 & 0 \\
0 & \frac{\partial N^{i}}{\partial x_{2}} & 0 \\
0 & 0 & \frac{\partial N^{i}}{\partial x_{3}} \\
\frac{\partial N^{i}}{\partial x_{2}} & \frac{\partial N^{i}}{\partial x_{1}} & 0 \\
\frac{\partial N^{i}}{\partial x_{3}} & 0 & \frac{\partial N^{i}}{\partial x_{1}} \\
0 & \frac{\partial N^{i}}{\partial x_{3}} & \frac{\partial N^{i}}{\partial x_{2}}
\end{array}\right], \quad \mathbf{D}=\mu\left(\left[\begin{array}{cc}
2 \mathbf{I}_{3} & \mathbf{0} \\
\mathbf{0} & \mathbf{I}_{3}
\end{array}\right]-\frac{2}{3} \mathbf{m m}^{\mathrm{T}}\right) \\
& \mathbf{I}_{3}=\left[\begin{array}{lll}
1 & 0 & 0 \\
0 & 1 & 0 \\
0 & 0 & 1
\end{array}\right], \quad \mathbf{f}_{i}=\int_{\Omega^{\mathrm{e}}} N^{i} \mathbf{b} \mathrm{d} \Omega+\int_{\Gamma^{\mathrm{e}}} N^{i} \mathbf{t} \mathrm{d} \Gamma
\end{aligned}
$$

where $i, j, k=1, n_{\mathrm{d}}$ in the above expressions.

A 3D finite element has typically 10 d.o.f.: three velocities $\bar{u}_{i}^{k}$, one pressure $\bar{p}^{k}$, three pressure gradient projections $\bar{\pi}_{c}^{k}$ and three convective projections $\bar{c}_{i}^{k}, i=1,2,3$. Note, however, that the solution for the $\bar{\pi}$ and $\overline{\mathbf{c}}$ variables is usually decoupled from the rest of equations and it is performed explicitly as shown in Section 8.

\section{TRANSIENT SOLUTION SCHEME}

The solution in time of the system of Equations (43) can be written in general form as

$$
\begin{gathered}
\mathbf{M} \frac{1}{\Delta t}\left(\overline{\mathbf{u}}^{n+1}-\overline{\mathbf{u}}^{n}\right)+\mathbf{H}^{n+\theta} \overline{\mathbf{u}}^{n+\theta}-\mathbf{G} \overline{\mathbf{p}}^{n+\theta}+\mathbf{C}^{n+\theta} \overline{\mathbf{c}}^{n+\theta}=\mathbf{f}^{n+\theta} \\
\mathbf{G}^{\mathrm{T}} \overline{\mathbf{u}}^{n+\theta}+\hat{\mathbf{L}}^{n+\theta} \overline{\mathbf{p}}^{n+\theta}+\mathbf{Q}^{n+\theta} \overline{\boldsymbol{\pi}}^{n+\theta}=\mathbf{0} \\
\hat{\mathbf{C}}^{n+\theta} \overline{\mathbf{u}}^{n+\theta}+\mathbf{M} \overline{\mathbf{c}}^{n+\theta}=\mathbf{0} \\
{\left[\mathbf{Q}^{n+\theta}\right]^{\mathrm{T}} \overline{\mathbf{p}}^{n+\theta}+\hat{\mathbf{M}}^{n+\theta} \bar{\pi}^{n+\theta}=\mathbf{0}}
\end{gathered}
$$

where $\mathbf{H}^{n+\theta}=\mathbf{H}\left(\overline{\mathbf{u}}^{n+\theta}\right)$, etc. and the parameter $\theta \in[0,1]$. The direct monolithic solution of Equations (45) is possible using an adequate iterative scheme. However, in our work we have used the fractional step method described next. 


\section{FRACTIONAL STEP METHOD}

A fractional step scheme is derived by splitting the discretized momentum equation (45a) into the following two equations:

$$
\begin{gathered}
\mathbf{M} \frac{1}{\Delta t}\left(\tilde{\mathbf{u}}^{n+1}-\overline{\mathbf{u}}^{n}\right)+\mathbf{H}^{n+\theta} \tilde{\mathbf{u}}^{n+\theta}-\alpha \mathbf{G} \overline{\mathbf{p}}^{n}+\mathbf{C}^{n+\theta} \overline{\mathbf{c}}^{n+\theta}=\mathbf{f}^{n+\theta} \\
\mathbf{M} \frac{1}{\Delta t}\left(\overline{\mathbf{u}}^{n+1}-\tilde{\mathbf{u}}^{n+1}\right)-\mathbf{G}\left(\overline{\mathbf{p}}^{n+1}-\alpha \overline{\mathbf{p}}^{n}\right)=\mathbf{0}
\end{gathered}
$$

In Equations (46) $\tilde{\mathbf{u}}^{n+1}$ is a predicted value of the velocity at time $n+1$ and $\alpha$ is a variable whose values of interest are zero and one. For $\alpha=0$ (first-order scheme) the splitting error is of order $0(\Delta t)$, whereas for $\alpha=1$ (second-order scheme) the error is of order $0\left(\Delta t^{2}\right)[19,21]$. We have chosen $\alpha=1$ for the solution of the examples presented in the paper.

Equations (46) are completed with the following three equations emanating from Equations (45b)-(45d)

$$
\begin{gathered}
\mathbf{G}^{\mathrm{T}} \overline{\mathbf{u}}^{n+1}+\hat{\mathbf{L}}^{n} \overline{\mathbf{p}}^{n+1}+\mathbf{Q}^{n} \bar{\pi}^{n}=\mathbf{0} \\
\hat{\mathbf{C}}^{n+1} \overline{\mathbf{u}}^{n+1}+\mathbf{M} \overline{\mathbf{c}}^{n+1}=\mathbf{0} \\
{\left[\mathbf{Q}^{n+1}\right]^{\mathrm{T}} \overline{\mathbf{p}}^{n+1}+\hat{\mathbf{M}}^{n+1} \bar{\pi}^{n+1}=\mathbf{0}}
\end{gathered}
$$

The value of $\overline{\mathbf{u}}^{n+1}$ obtained from Equation (47b) is substituted into Equation (47a) to give

$$
\mathbf{G}^{\mathrm{T}} \tilde{\mathbf{u}}^{n+1}+\Delta t \mathbf{G}^{\mathrm{T}} \mathbf{M}^{-1} \mathbf{G}\left(\overline{\mathbf{p}}^{n+1}-\alpha \overline{\mathbf{p}}^{n}\right)+\hat{\mathbf{L}}^{n} \mathbf{p}^{n+1}+\mathbf{Q}^{n} \bar{\pi}^{n}=\mathbf{0}
$$

The product $\mathbf{G}^{\mathrm{T}} \mathbf{M}^{-1} \mathbf{G}$ can be approximated by a Laplacian matrix, i.e.

$$
\mathbf{G}^{\mathrm{T}} \mathbf{M}^{-1} \mathbf{G}=\frac{1}{\rho} \mathbf{L} \quad \text { with } L_{i j}=\int_{\Omega^{\mathrm{e}}}\left(\nabla N^{i}\right)^{\mathrm{T}} \nabla N^{j} \mathrm{~d} \Omega
$$

The steps of the fractional step scheme (for $\alpha=1$ ) are as follows.

Step 1: Equation (46a) is linearized as

$$
\mathbf{M} \frac{\tilde{\mathbf{u}}^{n+1}-\overline{\mathbf{u}}^{n}}{\Delta t}+\mathbf{H}^{n} \overline{\mathbf{u}}^{n}-\mathbf{G} \overline{\mathbf{p}}^{n}+\mathbf{C}^{n} \overline{\mathbf{c}}^{n}=\mathbf{f}^{n}
$$

The fractional nodal velocities $\tilde{\mathbf{u}}^{n+1}$ can be explicitly computed from Equation (50) by

$$
\tilde{\mathbf{u}}^{n+1}=\overline{\mathbf{u}}^{n}-\Delta t \mathbf{M}_{\mathrm{d}}^{-1}\left[\mathbf{H}^{n} \overline{\mathbf{u}}^{n}-\mathbf{G} \overline{\mathbf{p}}^{n}+\mathbf{C}^{n} \overline{\mathbf{c}}^{n}-\mathbf{f}^{n}\right]
$$

Step 2: Compute $\overline{\mathbf{p}}^{n+1}$ from Equation (48) as

$$
\overline{\mathbf{p}}^{n+1}=-\left[\hat{\mathbf{L}}^{n}+\frac{\Delta t}{\rho} \mathbf{L}\right]^{-1}\left[\mathbf{G}^{\mathrm{T}} \tilde{\mathbf{u}}^{n+1}-\frac{\Delta t}{\rho} \mathbf{L} \overline{\mathbf{p}}^{n}+\mathbf{Q}^{n} \bar{\pi}^{n}\right]
$$

Step 3: Compute $\overline{\mathbf{u}}^{n+1}$ explicitly from Equation (46b) as

$$
\overline{\mathbf{u}}^{n+1}=\tilde{\mathbf{u}}^{n+1}+\Delta t \mathbf{M}_{\mathrm{d}}^{-1} \mathbf{G}\left(\overline{\mathbf{p}}^{n+1}-\overline{\mathbf{p}}^{n}\right)
$$


Step 4: Compute $\overline{\mathbf{c}}^{n+1}$ explicitly from Equation (47b) as

$$
\overline{\mathbf{c}}^{n+1}=-\mathbf{M}_{\mathrm{d}}^{-1} \hat{\mathbf{C}}^{n+1} \overline{\mathbf{u}}^{n+1}
$$

Step 5: Compute $\bar{\pi}^{n+1}$ explicitly from Equation (47c) as

$$
\bar{\pi}^{n+1}=-\hat{\mathbf{M}}_{\mathrm{d}}^{-1}\left[\mathbf{Q}^{n+1}\right]^{\mathrm{T}} \overline{\mathbf{p}}^{n+1}
$$

In the above equations $\mathbf{M}_{\mathrm{d}}$ and $\hat{\mathbf{M}}_{\mathrm{d}}$ denote the lumped diagonal form of matrices $\mathbf{M}$ and $\hat{\mathbf{M}}$, respectively.

Steps 1-5 are repeated until convergence for $\overline{\mathbf{u}}^{n+1}, \overline{\mathbf{p}}^{n+1}, \overline{\mathbf{c}}^{n+1}$ and $\bar{\pi}^{n+1}$ is found. Typically, three iterations per time step sufficed to find a converged solution in the examples presented in the paper.

Above algorithm has improved stabilization properties versus the standard pressure segregation methods due to the introduction of the Laplacian matrix $\hat{\mathbf{L}}$ in Equation (52) which emanates from the FIC stabilization terms.

The boundary conditions are applied as follows. No condition is applied in the computation of the fractional velocities $\tilde{\mathbf{u}}^{n+1}$ in Equation (51). The prescribed velocities at the boundary are applied when solving for $\overline{\mathbf{u}}^{n+1}$ in the Step 3. The prescribed pressures at the boundary are imposed by making $\overline{\mathbf{p}}^{n}$ equal to the pressure values computed explicitly from the Neumann boundary condition (19a), neglecting the stabilization terms, i.e.

$$
p^{n}=\frac{1}{3} \sum_{i}\left(s_{i j}^{n} n_{j}-t_{i}\right), \quad i=1, n_{\mathrm{d}}
$$

Equation (56) shows that for low values of the viscosity, the standard assumption of $p^{n}=0$ on free surfaces (with $t_{i}=0$ ) can be used.

We note that there is no need to prescribe any value of the pressure at the boundary if the form of matrix $\mathbf{L}=\rho \mathbf{G}^{\mathrm{T}} \mathbf{M}^{-1} \mathbf{G}$ as deduced from Equation (48) is used. This expression for $\mathbf{L}$ has a wider bandwidth than the Laplacian form of Equation (48) and therefore it is more inconvenient for practical purposes. In our work, we have used for $\mathbf{L}$ the simple Laplacian form of Equation (49).

\section{COMPUTATION OF THE CHARACTERISTIC DISTANCES}

The computation of the stabilization parameters is a crucial issue as they affect both the stability and accuracy of the numerical solution. The different procedures to compute the stabilization parameters are typically based on the study of simplified forms of the stabilized equations [1-28].

Recent work of the authors has shown that the stabilizing FIC terms for convection-diffusion problems take the form of a simple orthotropic diffusion if the balance equation is written in the principal curvature directions of the solution. Excellent results were reported in [45, 47] by computing first the characteristic length distances along the principal curvature directions, followed by a standard transformation of these distances to global axes. The resulting stabilized finite element equations capture the high gradient zones in the vicinity of the domain edges (boundary layers) as well as the sharp gradients appearing randomly in the interior of the domain [45,47]. The FIC/FEM thus reproduces the best features of the so-called transverse (cross-wind) dissipation or shock-capturing methods $[25,26]$. 


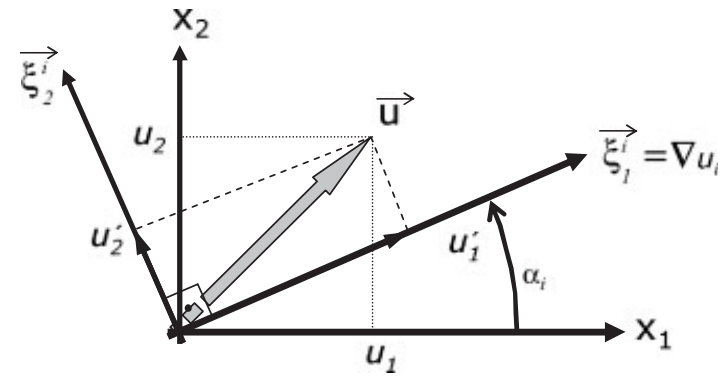

Figure 6. Definition of the principal curvature direction $\xi_{1}^{i}$ along the gradient of $u_{i}$.

Let us assume that there exists at each point a local orthogonal coordinate system characterized by the local directions $\xi_{j}^{i}$ with associated vectors $\xi_{j}^{i}(j=1,2$ for $2 \mathrm{D}$ problems $)$ such that $\partial^{2} u_{i}^{\prime} / \partial \xi_{j}^{i} \partial \xi_{k}^{i}=0$ for $j \neq k$, where $u_{i}^{\prime}$ is the velocity component along the $\xi_{i}^{i}$ direction (Figure 6). The $i$ th FIC momentum equation (23) written in such a local coordinate system reads

$$
\rho\left[\frac{\partial u_{i}^{\prime}}{\partial t}+u_{j}^{\prime} \frac{\partial u_{i}^{\prime}}{\partial \xi_{j}^{i}}\right]+\frac{\partial p}{\partial \xi_{j}^{i}}-\frac{\partial}{\partial \xi_{j}^{i}}\left(\mu+\bar{\mu}_{j j}\right) \frac{\partial u_{i}^{\prime}}{\partial \xi_{j}^{i}}-b_{i}-\frac{h_{i k}^{\prime}}{2} \frac{\partial c_{i}}{\partial \xi_{k}^{i}}+\mu \frac{\partial \varepsilon_{v}}{\partial \xi_{i}^{i}}=0
$$

The stabilizing dissipation introduced by the FIC approach has now the form of the orthotropic viscosity term underlined in Equation (57). For 2D problems

$$
\frac{\partial}{\partial \xi_{j}^{i}} \bar{\mu}_{j j} \frac{\partial u_{i}^{\prime}}{\partial \xi_{j}^{i}}=\frac{\partial}{\partial \xi_{1}^{i}}\left(\bar{\mu}_{11} \frac{\partial u_{i}^{\prime}}{\partial \xi_{1}^{i}}\right)+\frac{\partial}{\partial \xi_{2}^{i}}\left(\bar{\mu}_{22} \frac{\partial u_{i}^{\prime}}{\partial \xi_{2}^{i}}\right)
$$

with

$$
\bar{\mu}_{j j}=\frac{\rho u_{j}^{\prime} h_{i j}^{\prime}}{2} \text { no sum in } j
$$

The characteristic length distances $h_{i j}^{\prime}$ in Equation (57) are defined in the local axes $\xi_{j}^{i}$. Note that the upper index $i$ in vector $\xi_{j}^{i}$ denotes the $i$ th momentum equation corresponding to the $u_{i}^{\prime}$ velocity, while index $j$ denotes the local directions, i.e. $\xi_{1}^{i}, \xi_{2}^{i}$ are the two local coordinate directions corresponding to the $i$ th momentum equation (Figure 6).

The value of $h_{i j}^{\prime}$ can be estimated by analogy of Equation (57) with the linear 1D advectiondiffusion equation

$$
\rho u_{j}^{\prime} \frac{\partial \phi}{\partial \xi_{j}^{i}}-\frac{\partial}{\partial \xi_{j}^{i}}\left(\mu+\bar{\mu}_{j j}\right) \frac{\partial \phi}{\partial \xi_{j}^{i}}=0 \text { no sum in } i
$$

where $\phi$ is the transported variable. 


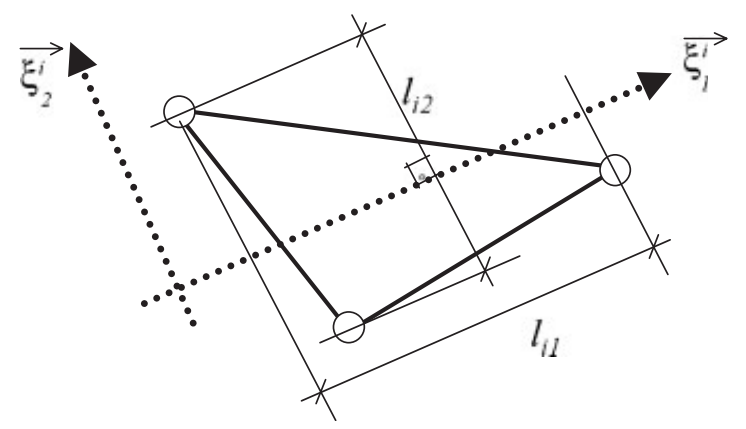

Figure 7. Definition of the element characteristic distances $l_{i 1}$ and $l_{i 2}$ corresponding to the $i$ th momentum equation.

Introducing into Equation (60) the expression of $\bar{\mu}_{j j}$ of Equation (59) and assuming $\mu$ and $\bar{\mu}_{j j}$ to be independent of the space coordinates gives (for $\phi=u_{i}^{\prime}$ )

$$
h_{i j}^{\prime}=\left[2\left(\frac{\partial u_{i}^{\prime}}{\partial \xi_{j}^{i}}\right)\left(\frac{\partial^{2} u_{i}^{\prime}}{\partial \xi_{j}^{i 2}}\right)^{-1}-\frac{1}{\gamma_{i j}}\right] l_{i j}=\alpha_{i j} l_{i j} \quad \text { no sum in } i, j
$$

where

$$
\alpha_{i j}=2\left(\frac{\partial u_{i}^{\prime}}{\partial \xi_{j}^{i}}\right)\left(\frac{\partial^{2} u_{i}^{\prime}}{\partial \xi_{j}^{i 2}}\right)^{-1}-\frac{1}{\gamma_{i j}}, \quad \gamma_{i j}=\frac{\rho u_{i}^{\prime} l_{i j}}{2 \mu}
$$

and $l_{i j}$ is a characteristic element dimension along the $\xi_{j}^{i}$ direction (Figure 7).

Note that $\gamma_{i j}$ can be interpreted as a local Reynolds number. It can be shown that $\alpha_{i j} \rightarrow 1$ for large values of $\gamma_{i j}$ inducing high local gradients of the transported variable.

A good approximation for $\alpha_{i j}$ deduced by analogy with the stabilization parameter for the linear advection-diffusion equation $[1,37,39]$ is

$$
\alpha_{i j}=\operatorname{coth} \gamma_{i j}-\frac{1}{\gamma_{i j}}
$$

Observation of Equation (63) shows that $\alpha_{i j}>0.95$ for $\gamma_{i j}>20$. Indeed, $\alpha_{i j} \simeq 1$ for high values of $\gamma_{i j}$ typical of turbulent flows.

The characteristic distances $h_{i j}$ are finally computed by transforming their local values $h_{i j}^{\prime}$ to global axes $x_{i}$. Details of the transformation are given below.

The numerical computations are simplified without apparent loss of accuracy if the $\xi_{1}^{i}$ direction is taken to be constant within each element and equal to the direction of the gradient of the $u_{i}$ velocity component at the element centre. The other coordinates $\xi_{j}^{i}(j=2,3$ for $3 \mathrm{D}$ problems $)$ are defined so as to form an orthogonal system with $\xi_{1}^{i}$.

The algorithm described above for computing the characteristic distances $h_{i j}$ is detailed below for 3D problems and linear tetrahedra elements. The particular form of some expressions for $2 \mathrm{D}$ problems using 3-noded linear triangles is given. 
For the $i$ th momentum balance equation and every time step of the transient solution scheme:

1. A coordinate system $\xi_{1}^{i}, \xi_{2}^{i}, \xi_{3}^{i}$ is defined at the element centre such that vector $\xi_{1}^{i}$ is aligned with the gradient of $u_{i}\left(\xi_{1}^{i}=\nabla u_{i}\right)$, vector $\xi_{2}^{i}$ is orthogonal to $\xi_{1}^{i}$ in anticlockwise sense and vector $\xi_{3}^{i}$ is defined by the vector product of $\xi_{1}^{i}$ and $\xi_{2}^{i}$. Figure 6 shows the definition of $\xi_{1}^{i}$ and $\xi_{2}^{i}$ for $2 \mathrm{D}$ problems.

2. The element characteristic distances $l_{i j}, j=1,2,3$ are defined as the maximum projections of the element sides along the $\xi_{j}^{i}$ axes (Figure 7).

3. The characteristic distances $h_{i j}, j=1,2,3$ are computed as

$$
\mathbf{h}_{i}=\mathbf{T h}_{i}^{\prime}, \quad i=1,2
$$

with

$$
\mathbf{T}=\left[\begin{array}{ccc}
\left(1,1^{\prime}\right)^{i} & \left(1,2^{\prime}\right)^{i} & \left(1,3^{\prime}\right)^{i} \\
\left(2,1^{\prime}\right)^{i} & \left(2,2^{\prime}\right)^{i} & \left(2,3^{\prime}\right)^{i} \\
\left(3,1^{\prime}\right)^{i} & \left(3,2^{\prime}\right)^{i} & \left(3,3^{\prime}\right)^{i}
\end{array}\right], \quad \mathbf{h}_{i}=\left\{\begin{array}{l}
h_{i 1} \\
h_{i 2} \\
h_{i 3}
\end{array}\right\}, \quad \mathbf{h}_{i}^{\prime}=\left\{\begin{array}{c}
h_{i 1}^{\prime} \\
h_{i 2}^{\prime} \\
h_{i 3}^{\prime}
\end{array}\right\}
$$

where $(j, k)^{i}$ is the cosine of the angle between the global $x_{j}$-axis and the $\xi_{k}^{i}$-axis. For 2D problems

$$
\mathbf{T}=\left[\begin{array}{cc}
c_{i} & -s_{i} \\
s_{i} & c_{i}
\end{array}\right]
$$

where $c_{i}=\cos \alpha_{i}, s_{i}=\sin \alpha_{i}$ and $\alpha_{i}$ is the angle that $\xi_{1}^{i}$ forms with the global axis $x_{1}$ (Figure 6). The local distances $h_{i j}^{\prime}$ are computed as

$$
h_{i j}^{\prime}=\left(\operatorname{coth} \gamma_{i j}-\frac{1}{\gamma_{i j}}\right) l_{i j}, \quad \gamma_{i j}=\frac{\rho u_{j}^{\prime} l_{i j}}{2 \mu}, \quad j=1,2
$$

where $u_{j}^{\prime}$ is the component of the velocity vector along the local axis $\xi_{j}^{i}$ (Figure 6).

\section{EXAMPLES}

The first version of the FIC/FEM stabilized formulation presented above was successfully tested in a number of $2 \mathrm{D}$ problems including the flow over a backwards facing step and the flow past a cylinder. Excellent results were obtained for a range of Reynolds numbers as reported in [35]. The first 3D application of a flow past a cylinder at $R e=1000$ also produced excellent results and it was briefly reported in [36]. The examples presented next provide further evidence of the effectiveness and accuracy of the FIC/FEM formulation presented in this paper for solving complex flows at high Reynolds numbers exhibiting turbulence effects. 


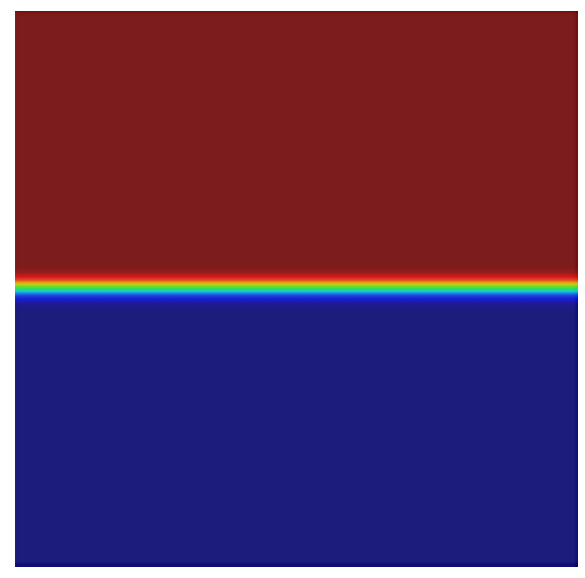

Figure 8. Initial configuration for the 2D mixing layer problem.

\section{1. $2 D$ mixing layer}

We consider a temporal developing mixing layer [59], schematically sketched in Figure 8. The initial horizontal velocity has a hyperbolic-tangent profile:

$$
u_{1}\left(x_{2}\right)=U \tanh \left(\frac{2 x_{2}}{\delta_{0}}\right)
$$

which implies a vorticity thickness

$$
\delta_{0}=\frac{2 U}{\left.\frac{\mathrm{d} u_{1}}{\mathrm{~d} x_{2}}\right|_{x_{2}=0}}
$$

From linear stability analysis the mixing layer is known to be invisicidly unstable. A perturbation leads to the formation of vortices by Kelvin-Helmholtz instability, where the most amplified mode corresponds to a longitude wavelength $\delta=7 \delta_{0}$ [60]. Kelvin-Helmholtz instability leads to the development of vortices which in a later stage roll-up and merge.

The initial vorticity thickness $\delta_{0}$ is chosen such that four vortices should develop in a square domain of unit size. In order to triggering the instability we superimposed a weak white noise in the rotational region. The value of $U=1$ is chosen in Equation (68) and the viscosity is $\mu=3.571 \times 10^{-6}$ (given a Reynolds number of $2.8 \times 10^{5}$ ). The boundary conditions applied are: periodic boundary conditions on the lateral boundaries and zero-normal-velocity and zero-shearstress at the upper and bottom boundaries. With these boundary conditions, the problem is solved in a cylindrical domain.

We use a structured mesh of $256 \times 2563$-noded triangular elements. Five hundred and seventy time steps of $0.0125 \mathrm{~s}$ lead to a total simulation time of $7.125 \mathrm{~s}$.

The mixing layer is a good example for the tendency of 2D turbulence to transfer energy from small to large scales. This leads to a fast decrease of the complexity of the flow. In Figure 9 the vorticity modulus contours at several time steps is shown. Four vortices are formed as predicted by the linear theory, which subsequently undergo successive mergings. 


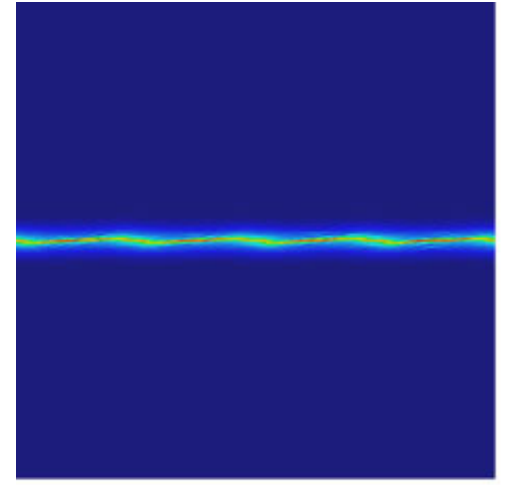

(a)

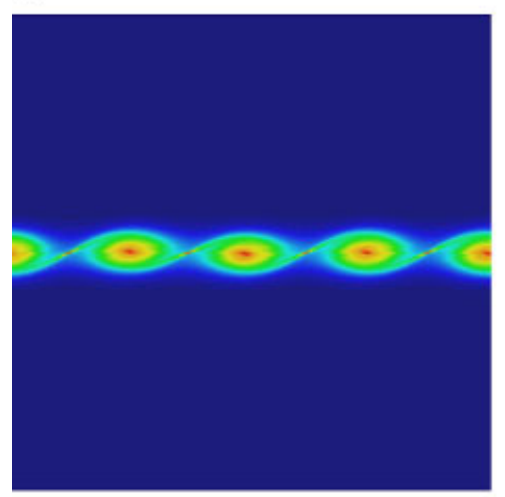

(c)

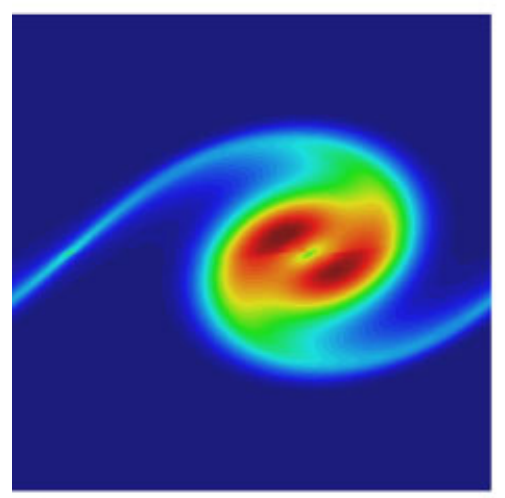

(e)

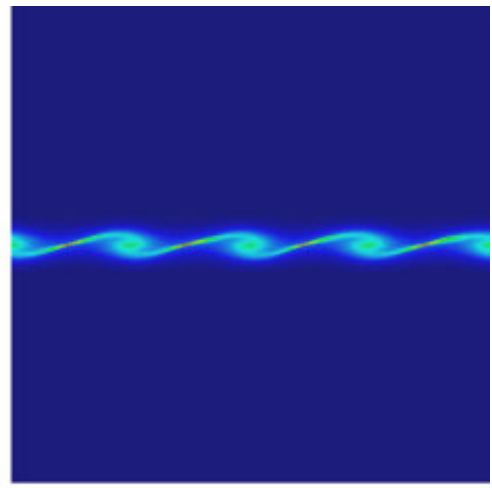

(b)

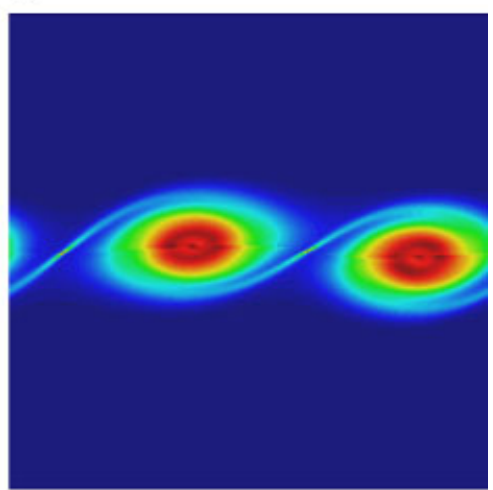

(d)

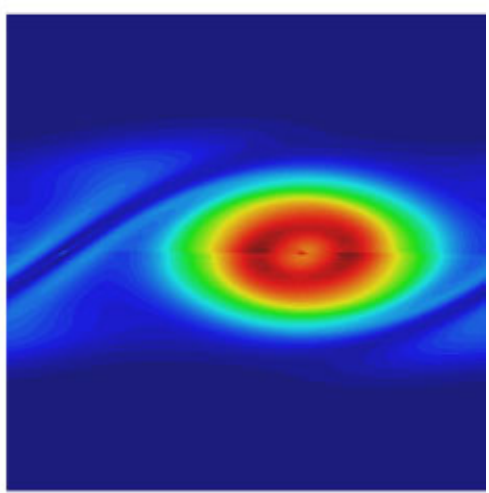

(f)

Figure 9. Vorticity modulus contours at times: (a) 0.5 ; (b) 0.75 ; (c) 1 ; (d) 2 ; (e) 3 ; and (f) 4 s.

For the reference simulations a Fourier spectral code was applied to the periodized version of the problem [61]. The code is based on the pressure-velocity formulation and uses a third order Adams-Bashforth (AB3) scheme. The numerical resolution was a grid of $256 \times 256$ too. Figure 10 


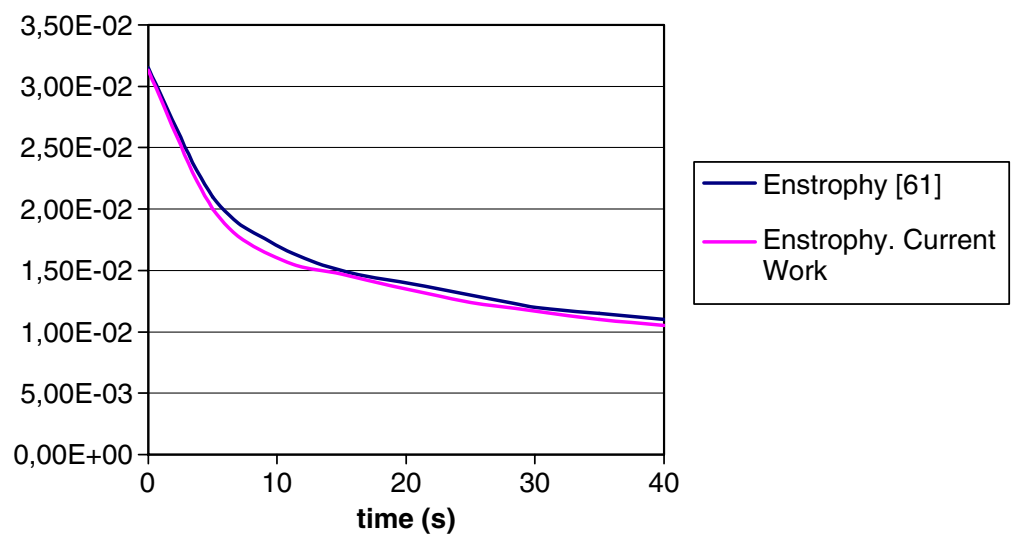

Figure 10. Enstrophy evolution with time.

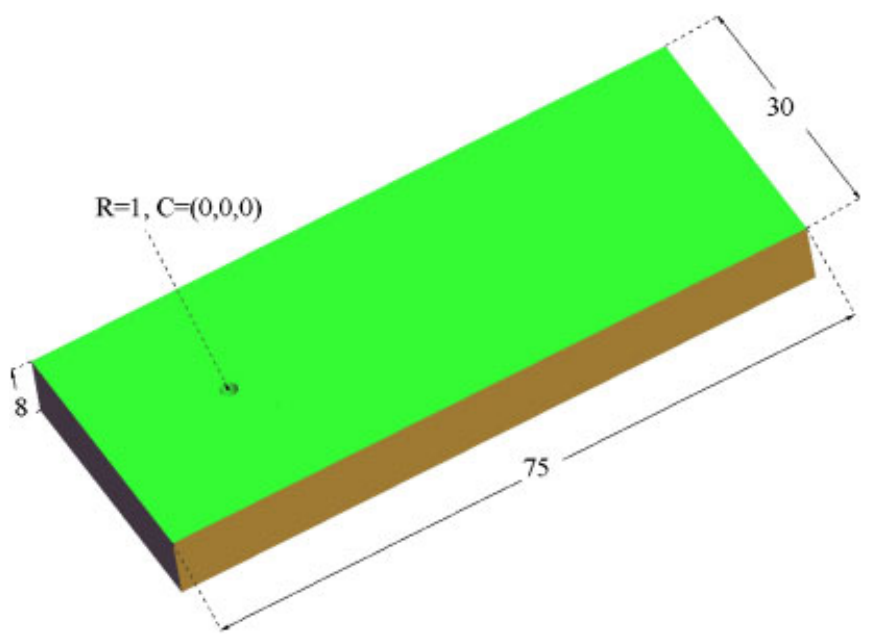

Figure 11. Computational domain for 3D flow past a cylinder.

compares the decay in time of the system enstrophy obtained with the reference run and with the present method. The results show that all the scales of the flow are well-resolved by the FIC formulation here proposed and compare well with the reference run.

\section{2. $3 D$ flow past a cylinder}

We present a 3D simulation of unsteady incompressible flow around a circular cylinder. The simulation is performed at a Reynolds number of 10000 .

The diameter of the cylinder is 2 units and its length is 8 units (this length is recommended in [62] to capture a few wavelengths along the cylinder axis). The computation domain extends 15 units upstream, 60 units downstream, and 30 units in the cross-flow direction (Figure 11). 

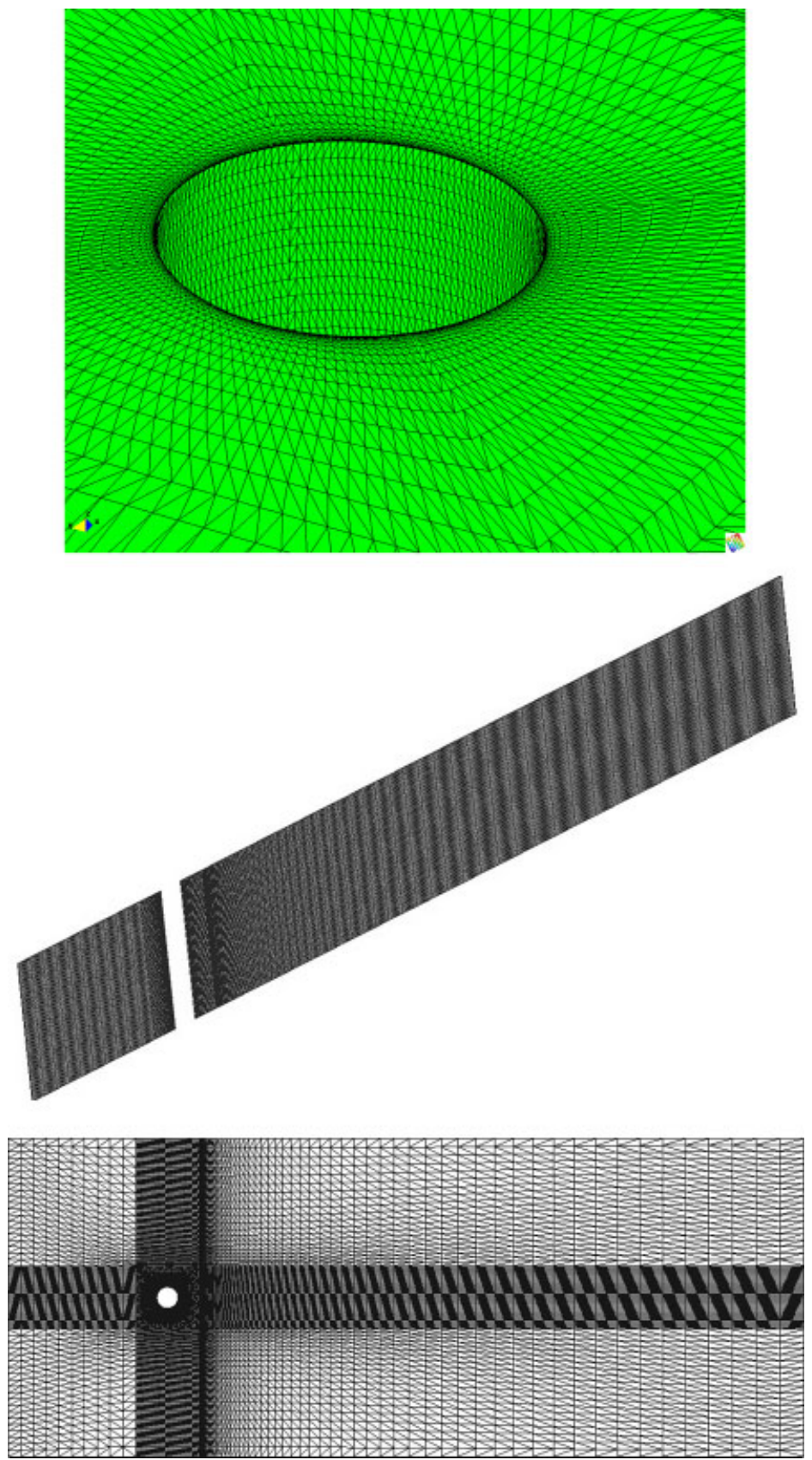

Figure 12. Flow past a cylinder. Details of the mesh used for the computations. 

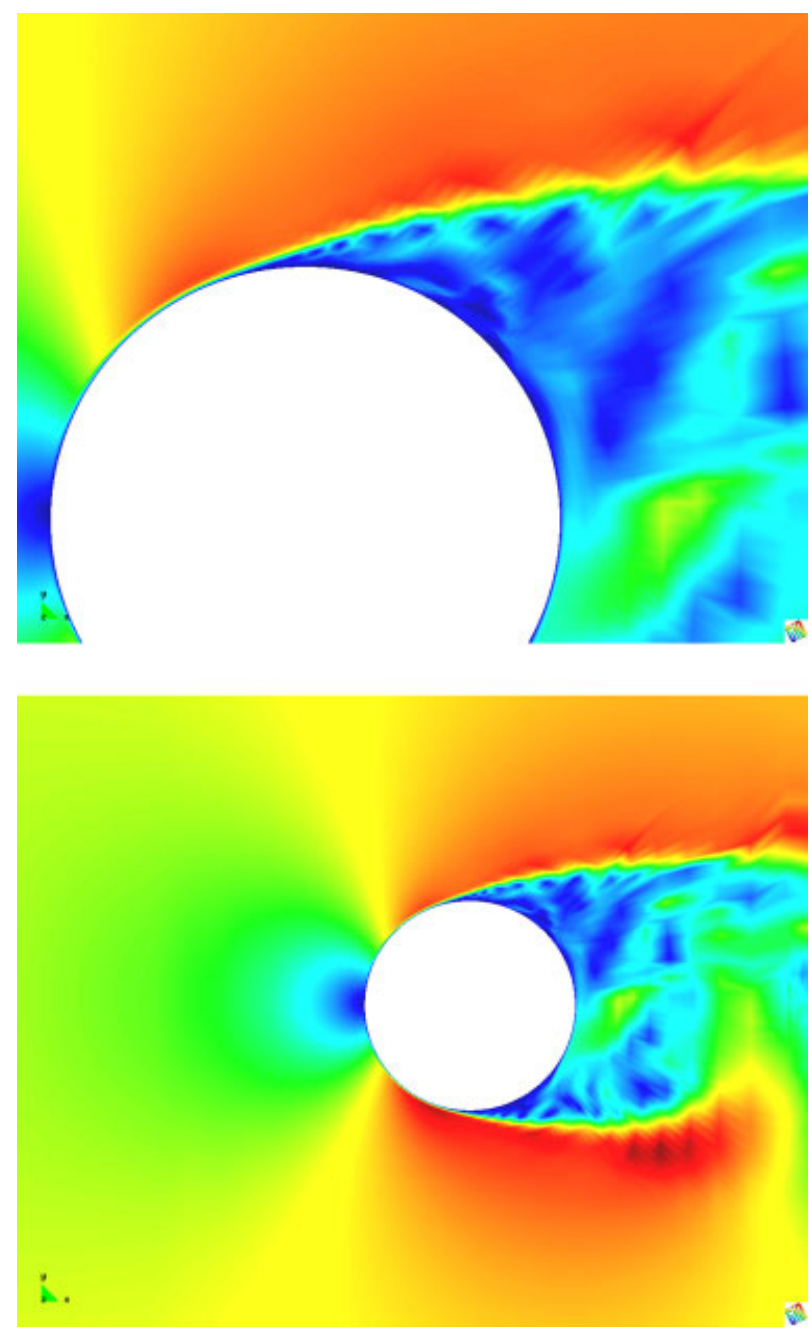

Figure 13. Velocity vector modulus contours in the plane $z=4$ at time $50 \mathrm{~s}$.

The boundary conditions consist of uniform inflow velocity set to 1.0, zero-normal-velocity and zero-shear-stress at the lateral boundaries, traction-free conditions at the outflow boundary and no-slip at the cylinder surface.

The computation presented here was carried out on a structured mesh of 5193600 linear tetrahedral elements (80 elements along the cylinder span and 160 along its circumference) and 864270 nodes. The thickness of the layer of elements around the cylinder is 0.001 . Figure 12 shows details of the mesh. For the simulation the time step is set to 0.025 . The time-averaged drag coefficient is 1.07 and compares well with the value of 1.12 reported in experimental measurement $[63,64]$. The Strouhal number is 2.02 and also agrees with experimental measurements $[36,38,64]$. 


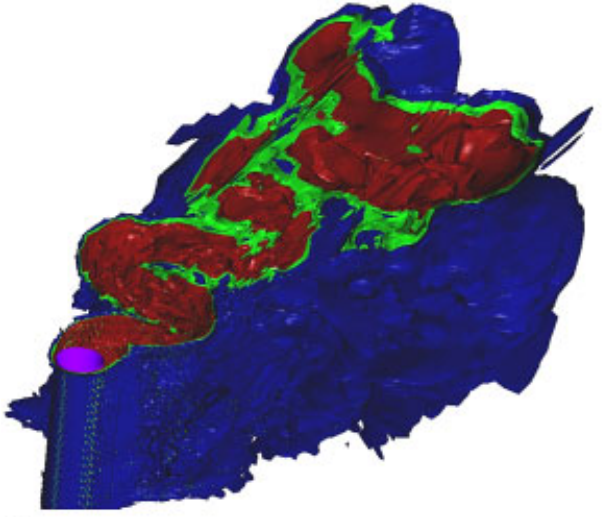

(a)

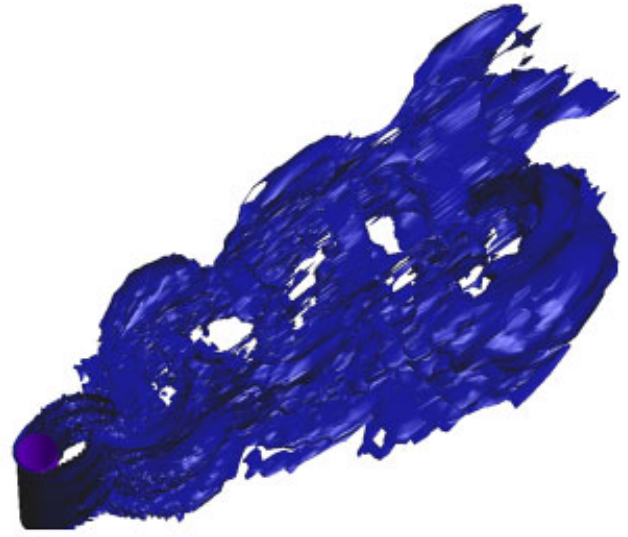

(b)

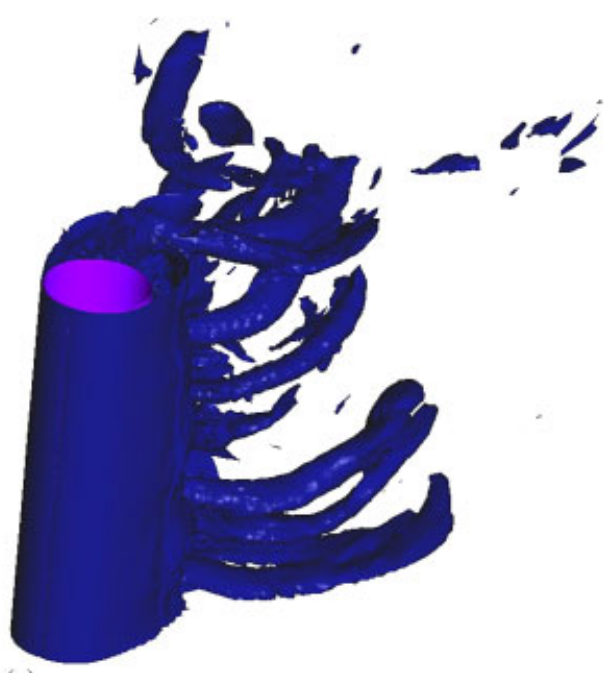

(c)

Figure 14 . Vorticity vector modulus $\omega$ isosurfaces: (a) $\omega=0.1,0.2,0.3$; (b) $\omega=0.2$; and (c) $\omega=2$.

The flow field in chordwise planes (perpendicular to the cylinder axis) reveals fine-scale structures. There is a clear difference between the turbulent wake and the laminar outer flow zones (see Figure 13). We observe the turbulent recirculating region bounded by shear layers. The shear layers roll up to produce small-scale vortices at the edge of the formation zone. These vortices cause entrainment of the free-stream fluid into the recirculating zone. The flow on the cylinder separates at an angle $\approx 78^{\circ}$ (measured from the leading stagnation point).

Figure 14 shows the isosurfaces of the vorticity vector modulus for three different vorticity values. Note that the flow structures are more diffuse due to the increasing turbulence effect.

Figure 15 shows streamlines behind the cylinder within the recirculation area. It is clear that the structure of the vortex is created in the turbulent region. When the vortex gets enough energy then it detaches from the cylinder, generating the von Karman street vortexes. 

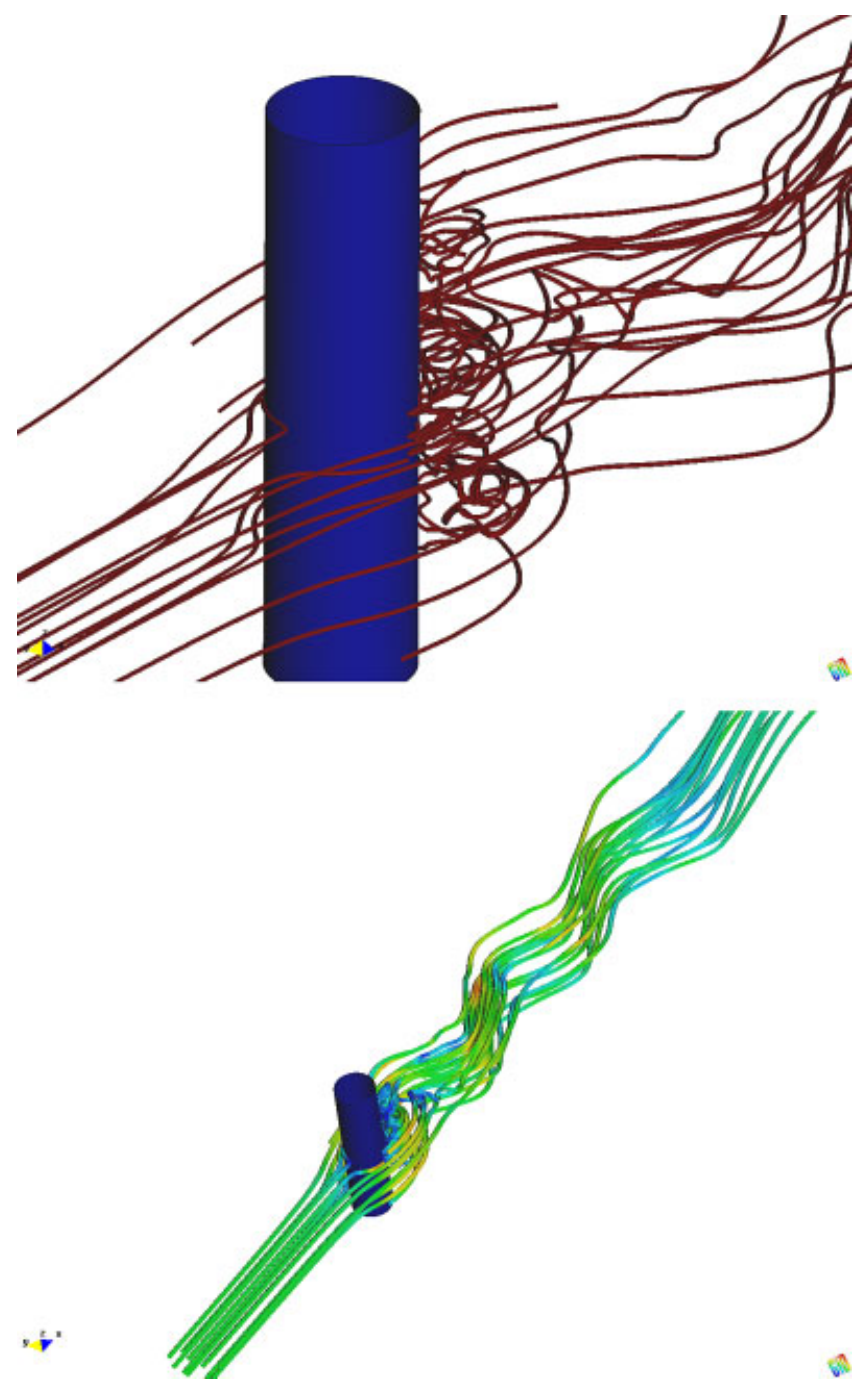

Figure 15. Streamlines at time $t=50$.

\section{CONCLUSIONS}

The finite calculus (FIC) form of the Navier-Stokes equations is a good starting point for deriving stabilized FEM for solving a variety of incompressible fluid flow problems. The matrix stabilization terms introduced by the FIC formulation allow to obtaining physically sound solutions in the presence of sharp gradients occurring for high Reynolds numbers without the need of introducing a turbulence model. Good numerical solutions have been obtained in the 2D and 3D examples solved with relatively coarse meshes for high values of the Reynolds number inducing turbulence effects. These results reinforce our conviction that the stabilization terms introduced by the FIC formulation suffice to provide good results for problems for which turbulence models are required 
using alternative numerical methods. The results also confirm the close link between the stabilized methods and turbulence models.

\section{ACKNOWLEDGEMENTS}

The authors are grateful to Prof. S. R. Idelsohn for many useful suggestions. Thanks are also given to the company COMPASS Ingeniería y Sistemas SA (www.compassis.com) for providing the code Tdyn [65] where the presented formulation has been implemented.

This research was partially supported by project SEDUREC of the Consolider Programme of the Ministerio de Educación y Ciencia of Spain.

\section{REFERENCES}

1. Heinrich JC, Hayakorn PS, Zienkiewicz OC. An upwind finite element scheme for two dimensional convective transport equations. International Journal for Numerical Methods in Engineering 1977; 11:131-143.

2. Kelly DW, Nakazawa S, Zienkiewicz OC. A note on anisotropic balancing dissipation in the finite element method approximation to convective diffusion problems. International Journal for Numerical Methods in Engineering 1980; 15:1705-1711.

3. Brooks AN, Hughes TJR. Streamline upwind Petrov-Galerkin formulation for convective dominated flows with particular emphasis on the incompressible Navier-Stokes equations. Computer Methods in Applied Mechanics and Engineering 1982; 32:199-259.

4. Donea J. A Taylor-Galerkin method for convective transport problems. International Journal for Numerical Methods in Engineering 1984; 20:101-119.

5. Löhner R, Morgan K, Zienkiewicz OC. The solution of non-linear hyperbolic equation systems by the finite element method. International Journal for Numerical Methods in Fluids 1984; 4:1043-1063.

6. Hughes TJR, Tezduyar TE. Finite element methods for first-order hyperbolic systems with particular emphasis on the compressible Euler equations. Computer Methods in Applied Mechanics and Engineering 1984; 45:217-284.

7. Hughes TJR, Mallet M. A new finite element formulations for computational fluid dynamics. III. The generalized streamline operator for multidimensional advective-diffusive systems. Computer Methods in Applied Mechanics and Engineering 1986; 58:305-328.

8. Hughes TJR, Franca LP, Balestra M. A new finite element formulation for computational fluid dynamics. V. Circumventing the Babuska-Brezzi condition: a stable Petrov-Galerkin formulation of the Stokes problem accommodating equal order interpolations. Computer Methods in Applied Mechanics and Engineering 1986; 59: 85-99.

9. Hughes TJR, Franca LP, Hulbert GM. A new finite element formulation for computational fluid dynamics. VIII. The Galerkin/least-squares method for advective-diffusive equations. Computer Methods in Applied Mechanics and Engineering 1989; 73:173-189.

10. Hirsch C. Numerical Computation of Internal and External Flow, vol. 1. Wiley: New York, 1990.

11. Franca LP, Frey SL. Stabilized finite element methods. II. The incompressible Navier-Stokes equations. Computer Methods in Applied Mechanics and Engineering 1992; 99:209-233.

12. Tezduyar TE, Mittal S, Ray SE, Shih R. Incompressible flow computations with stabilized bilinear and linear equal order interpolation velocity-pressure elements. Computer Methods in Applied Mechanics and Engineering 1992; 95:221-242.

13. Hughes TJR, Hauke G, Jansenn K. Stabilized finite element methods in fluids: inspirations, origins, status and recent developments. In Recent Developments in Finite Element Analysis, Taylor RL, Hughes TJR, Oñate E, Zienkiewicz OC (eds). International Center for Numerical Methods in Engineering (CIMNE): Barcelona, Spain, 1994; 272-292.

14. Hughes TJR. Multiscale phenomena: Green functions, subgrid scale models, bubbles and the origins of stabilized methods. Computer Methods in Applied Mechanics and Engineering 1995; 127:387-401.

15. Storti M, Nigro N, Idelsohn SR. Steady state incompressible flows using explicit schemes with an optimal local preconditioning. Computer Methods in Applied Mechanics and Engineering 1995; 124:231-252.

16. Cruchaga MA, Oñate E. A finite element formulation for incompressible flow problems using a generalized streamline operator. Computer Methods in Applied Mechanics and Engineering 1997; 143:49-67. 
17. Codina R, Vazquez M, Zienkiewicz OC. A general algorithm for compressible and incompressible flow-Part III. The semi-implicit form. International Journal for Numerical Methods in Fluids 1998; 27:13-32.

18. Cruchaga MA, Oñate E. A generalized streamline finite element approach for the analysis of incompressible flow problems including moving surfaces. Computer Methods in Applied Mechanics and Engineering 1999; 173:241-255.

19. Codina R, Blasco J. Stabilized finite element method for the transient Navier-Stokes equations based on a pressure gradient operator. Computer Methods in Applied Mechanics and Engineering 2000; 182:277-301.

20. Tezduyar TE, Osawa Y. Finite element stabilization parameters computed from element matrices and vectors. Computer Methods in Applied Mechanics and Engineering 2000; 190:411-430.

21. Codina R, Zienkiewicz OC. CBS versus GLS stabilization of the incompressible Navier-Stokes equations and the role of the time step as stabilization parameter. Communications in Numerical Methods in Engineering 2002; 18(2):99-112.

22. Codina R. Stabilized finite element approximation of transient incompressible flows using orthogonal subscales. Computer Methods in Applied Mechanics and Engineering 2002; 191:4295-4321.

23. Nithiarasu P. An efficient artificial compressibility (AC) scheme based on the characteristic based split (CBS) method for incompressible flows. International Journal for Numerical Methods in Engineering 2003; 56: 1815-1845.

24. Tezduyar TE. Computation of moving boundaries and interfaces and stabilization parameters. International Journal for Numerical Methods in Fluids 2003; 43:555-575.

25. Donea J, Huerta A. Finite Element Method for Flow Problems. Wiley: New York, 2003.

26. Zienkiewicz OC, Taylor RL, Nietharasu P. The Finite Element Method, vol. 3. Elsevier: Amsterdam, 2005.

27. Tezduyar TE, Sathe S. Enhanced-approximation linear solution technique (EALST). Computer Methods in Applied Mechanics and Engineering 2004; 193:2033-2049.

28. Tezduyar TE, Sathe S. Enhanced-discretization successive update method (EDSUM). International Journal for Numerical Methods in Fluids 2005; 47:633-654.

29. Dubois T, Jauberteau F, Temam R. Incremental unknowns, multilevel methods and the numerical simulation of turbulence. Computer Methods in Applied Mechanics and Engineering 1998; 159:123-189.

30. Dubois T, Jauberteau F, Temam R. Dynamic Multilevel Methods and the Numerical Simulation of Turbulence. Cambridge University Press: Cambridge, 1999.

31. Hughes TJR, Mazzei L, Jansen KE. Large eddy simulation and the variational multiscale method. Computing and Visualization in Science 2000; 3:47-59.

32. Hughes TJR, Mazzei L, Oberai AA, Wray AA. The multiscale formulation of large eddy simulation: decay of homogeneous isotropic turbulence. Physics of Fluids 2001; 13:505-512.

33. Hughes TJR, Oberai AA, Mazzei L. Large eddy simulation of turbulent channel flows by the variational multiscale method. Physics of Fluids 2001; 13(6):1784-1799.

34. Hoffman J, Johnson C. A new approach to computational turbulence modeling. Computer Methods in Applied Mechanics and Engineering 2006; 195:2865-2880.

35. Oñate E, Valls A, García J. FIC/FEM formulation with matrix stabilizing terms for incompressible flows at low and high Reynolds numbers. Computational Mechanics 2006; 38(4-5):440-455.

36. Oñate E, Valls A, García J. Modeling incompressible flows at low and high Reynolds numbers via a finite calculus finite element approach. Journal of Computational Physics 2006, submitted.

37. Oñate E. Derivation of stabilized equations for advective-diffusive transport and fluid flow problems. Computer Methods in Applied Mechanics and Engineering 1998; 151:233-267.

38. Oñate E. A stabilized finite element method for incompressible viscous flows using a finite increment calculus formulation. Computer Methods in Applied Mechanics and Engineering 2000; 182(1-2):355-370.

39. Oñate E. Possibilities of finite calculus in computational mechanics. International Journal for Numerical Methods in Engineering 2004; 60(1):255-281.

40. Oñate E, García J, Idelsohn SR. Computation of the stabilization parameter for the finite element solution of advective-diffusive problems. International Journal for Numerical Methods in Fluids 1997; 25:1385-1407.

41. Oñate E, García J, Idelsohn SR. An alpha-adaptive approach for stabilized finite element solution of advectivediffusive problems with sharp gradients. In New Advances in Adaptive Computational Methods in Mechanics, Ladeveze P, Oden JT (eds). Elsevier: Amsterdam, 1998.

42. Oñate E, Manzan M. A general procedure for deriving stabilized space-time finite element methods for advectivediffusive problems. International Journal for Numerical Methods in Fluids 1999; 31:203-221. 
43. Oñate E, Manzan M. Stabilization techniques for finite element analysis of convection-diffusion problems. In Convection Heat Transfer, Sunden B, Comini G (eds). WIT Press: Southampton, U.K., 2000; Research Report PI183, CIMNE, Barcelona, 2000.

44. Oñate E. Multiscale computational analysis in mechanics using finite calculus: an introduction. Computer Methods in Applied Mechanics and Engineering 2003; 192(28-30):3043-3059.

45. Oñate E, Zárate F, Idelsohn SR. Finite element formulation for convective-diffusive problems with sharp gradients using finite calculus. Computer Methods in Applied Mechanics and Engineering 2006; 195:1793-1825.

46. Oñate E, Miquel J, Hauke G. Stabilized formulation for the advection-diffusion-absorption equation using finite calculus and linear finite elements. Computer Methods in Applied Mechanics and Engineering 2006; 195(33-36):3926-3946.

47. Oñate E, Miquel J, Zárate F. Stabilized solution of the multidimensional advection-diffusion-absorption equation using linear finite elements. Computers and Fluids 2007; 36(1):92-112.

48. Felippa CA, Oñate E. Nodally exact Ritz discretizations of 1D diffusion-absorption and Helmholtz equations by variational FIC and modified equation methods. Computational Mechanics 2007; 39(2):91-111.

49. Oñate E, García J. A finite element method for fluid-structure interaction with surface waves using a finite calculus formulation. Computer Methods in Applied Mechanics and Engineering 2001; 191:635-660.

50. Idelsohn SR, Oñate E, Del Pin F. A lagrangian meshless finite element method applied to fluid-structure interaction problems. Computers and Structures 2003; 81:655-671.

51. Oñate E, Idelsohn SR, Del Pin F, Aubry R. The particle finite element method. An overview. International Journal of Computational Methods 2004; 1(2):267-307.

52. Idelsohn SR, Oñate E, Del Pin F. The particle finite element method: a powerful tool to solve incompressible flows with free-surfaces and breaking waves. International Journal for Numerical Methods in Engineering 2004; 61:964-989.

53. Oñate E, García J, Idelsohn SR. Ship hydrodynamics. In Encyclopedia of Computational Mechanics, Stein E, de Borst R, Hughes TJR (eds), vol. 3, Chapter 18. Wiley: New York, 2004; 579-607.

54. Oñate E, García J, Idelsohn SR, Del Pin F. FIC formulations for finite element analysis of incompressible flows. Eulerian, ALE and Lagrangian approaches. Computer Methods in Applied Mechanics and Engineering 2006; 195(23-24):3001-3037.

55. Oñate E, Rojek J, Taylor RL, Zienkiewicz OC. Finite calculus formulation for incompressible solids using linear triangles and tetrahedra. International Journal for Numerical Methods in Engineering 2004; 59:1473-1500.

56. Oñate E, Taylor RL, Zienkiewicz OC, Rojek J. Aresidual correction method based on finite calculus. Engineering Computations 2003; 20(5/6):629-658.

57. Rojek J, Oñate E, Taylor RL. CBS-based stabilization in explicit solid dynamics. International Journal for Numerical Methods in Engineering 2006; 66:1547-1568.

58. Limache A, Idelsohn SR, Rossi R, Oñate E. The violation of objectivity in Laplace formulations of the Navier-Stokes equations. International Journal for Numerical Methods of Fluids 2007, submitted.

59. Lesieur M, Staquet C, Le Roy P, Comte P. The mixing layer and its coherence examined from the point of view of two dimensional turbulence. Journal of Fluid Mechanics 1998; 192:511-535.

60. Michalke A. On the inviscid instability of the hyperbolic tangent velocity profile. Journal of Fluid Mechanics 1964; 19:543-556.

61. Griebel M, Koster F. Adaptive wavelet solvers for the unsteady incompressible Navier-Stokes equations. In Advances in Mathematical Fluid Mechanics, Malek J, Rokyta M (eds). Springer: Berlin, 2000.

62. Kalro V, Tezduyar TE. Parallel 3D computation of unsteady flows around circular cylinders. Parallel Computing 1997; 23:1235-1248.

63. Schlichting H. Boundary-Layer Theory (7th edn). McGraw-Hill: New York, 1979.

64. Gerard JH. An experimental investigation of the oscillating lift and drag of a circular cylinder shedding turbulent vortices. Journal of Fluid Mechanics 1961; 11:244-256.

65. Tdyn. A Finite Element Code for Fluid-Dynamic Analysis. COMPASS Ingeniería y Sistemas SA, 2007 (Available from: www.compassis.com). 